September EMC'reviewMar15b

15

FILE

International Journal of Modern Physics E (c) World Scientific Publishing Company

\title{
The EMC Effect and High Momentum Nucleons in Nuclei
}

\author{
Or Hen \\ Tel Aviv University, Tel Aviv 69978, Israel \\ or.chen@mail.huji.ac.il \\ Douglas W. Higinbotham \\ Thomas Jefferson National Accelerator Facility, Newport News, Virginia 23606, USA \\ doug@jlab.org \\ Gerald A. Miller \\ Department of Physics, University of Washington, Seattle, WA 98195-1560, USA \\ miller@phys.washington.edu \\ Eli Piasetzky \\ Tel Aviv University, Tel Aviv 69978, Israel \\ eip@tauphy.tau.ac.il \\ Lawrence B. Weinstein \\ Old Dominion University, Norfolk, Virginia 23529, USA \\ lweinste@odu.edu \\ Received 1/4/2013
}

\begin{abstract}
Recent developments in understanding the influence of the nucleus on deep-inelastic structure functions, the EMC effect, are reviewed. A new data base which expresses ratios of structure functions in terms of the Bjorken variable $x_{A}=A Q^{2} /\left(2 M_{A} q_{0}\right)$ is presented. Information about two-nucleon short-range correlations from experiments is also discussed and the remarkable linear relation between short-range correlations and teh EMC effect is reviewed. A convolution model that relates the underlying source of the EMC effect to modification of either the mean-field nucleons or the short-range correlated nucleons is presented. It is shown that both approaches are equally successful in describing the current EMC data.
\end{abstract}

Keywords: EMC;SRC;2N-SRC;High Momentum Nucleons

\section{Introduction}

Basic models of nuclear physics describe the nucleus as a collection of free nucleons moving non-relativistically under the influence of the sum of two-nucleon forces, which can be treated approximately as a mean field. In this picture, in the rest frame of the nucleon, the partonic structure functions of bound and free nucleons should be identical. Therefore, it was generally expected that, except for nucleon Fermi 
motion effects, Deep Inelastic Scattering (DIS) experiments which are sensitive to the partonic structure function of the nucleon would give the same result for all nuclei.

Instead, the measurements show a reduction in the structure function of nucleons bound in nuclei relative to nucleons bound in deuterium - the EMC effect. Since its discovery, over 30 years ago, a large experimental and theoretical effort has been put into understanding the origin of the effect. While theorists have had no difficulty in creating models that qualitatively reproduce nuclear DIS data by itself, there is no generally accepted model. This is because the models are either not consistent with or do not attempt to explain other nuclear phenomena. The use of most modern models shows that while traditional nuclear effects such as binding and Fermi motion contribute to the EMC effect, modification of the bound nucleon structure is also required.

Studies of the effects of the many-body nucleon-nucleon interaction on the structure of the nucleus predict the existence of Short-Range Correlated (SRC) pairs. These are pairs of nucleons at short distance whose wave functions strongly overlap, giving them large relative momentum and low center of mass momentum, where high and low is relative to the Fermi momentum $\left(k_{F}\right)$ of the nucleus. Recent studies show that the magnitude of the EMC effect in any nucleus is linearly related to the number of two-nucleon SRC pairs in that nucleus. The observation of this phenomenological relationship raises a question of whether the medium modification of the nucleon structure is related to the nuclear mean field or to the SRC pairs. The answer to this question will give new insight regarding the origin of the EMC effect.

Sections 2 and 3 review the EMC and SRC research history respectively. Section 2.3 presents a new formalism to correct the measured EMC data for the difference in the definition of the Bjorken scaling variable for different nuclei (the corrected EMC data is presented in Appendix A. Section 4 presents the EMCSRC correlation, its implications, and a simple convolution model which compares treatments of the EMC effect based on nucleon modification occurring in SRC pairs with that based on nucleon modification occurring due to the mean field. Section 5 summarizes the paper.

\section{The Nuclear EMC Effect}

\subsection{Historical Overview}

Unpolarized inclusive lepton scattering depends on two independent variables that can be chosen as the negative of the square of the transferred four-momentum, $Q^{2}=-q^{2}$ and the Bjorken scaling variable for a proton $x_{p}=Q^{2} /\left(2 m_{p} \omega\right),-$ commonly noted as $x_{B}$ - where $m_{p}$ is the proton mass and $\omega$ the transferred energy in the proton rest frame. In Deep Inelastic Scattering (DIS), the momentum transfer is large $\left(Q^{2}>2(\mathrm{GeV} / \mathrm{c})^{2}\right)$ and the invariant mass of the transferred photon plus the target nucleon is greater than the masses of individual nucleon resonances, 
$W>2 \mathrm{GeV}$. This allows a measurement of the proton's inelastic structure function, $F_{2}^{p}\left(x_{p}, Q^{2}\right)$, which gives the weighted average of the proton quark distribution function:

$$
F_{2}^{p}\left(x_{p}, Q^{2}\right)=x_{p} \sum_{q} e_{q}^{2} \cdot\left(q^{p}\left(x_{p}\right)+\bar{q}^{p}\left(x_{p}\right)\right),
$$

where $q^{p}\left(x_{p}\right)$ and $\bar{q}^{p}\left(x_{p}\right)$ are the proton's quark and anti-quark distribution functions respectively, $e_{q}$ is the electric charge of the quark, and the sum runs over $q$ the different proton quark flavours (i.e. $u, d$, and $s$ ). The neutron inelastic structure function, $F_{2}^{n}\left(x_{p}, Q^{2}\right)$, is given by substituting in Eq. 1 the proton quark distributions by that of the neutron. The latter can be expressed using the proton distribution, assuming isospin (charge) symmetry (i.e. $u^{n}\left(x_{p}\right)=d^{p}\left(x_{p}\right), d^{n}\left(x_{p}\right)=u^{p}\left(x_{p}\right)$ etc.).

In the early 1980's, CERN's European Muon Collaboration (EMC) measured the per-nucleon DIS cross section for scattering unpolarized muons from deuterium and iron nuclei and extracted the ratio of their structure functions ${ }^{1}$ (i.e., $\left.F_{2}^{F e}\left(x_{p}, Q^{2}\right) / F_{2}^{d}\left(x_{p}, Q^{2}\right)\right)$. The latter are the average bound nucleon structure function in ${ }^{56} \mathrm{Fe}$ and Deuterium. For $x_{p} \leq 0.5 \sim 0.7$, where nucleon Fermi motion effects are negligible, they expected to measure a ratio of unity, indicating that the structure function of deeply bound (i.e. ${ }^{56} \mathrm{Fe}$ ) and loosely bound (i.e. Deuterium) nucleons is identical. This would allow them to increase the experimental luminosity by using a denser target material such as iron, while still being sensitive to the free nucleon structure function. Instead, they discovered that the per-nucleon DIS cross section ratio, which equals the structure function ratio, decreased from about 1 at $x_{p} \approx 0.3$ to as little as 0.8 at $x_{p} \approx 0.7$ (see Fig. 1). This unexpected result instantly became known as the EMC effect. The existence of the EMC effect was soon verified by analysis of existing target end-cap data from SLAC, [2] and later by measurements at SLAC ${ }^{4}$ and the BCDMS and NMC collaborations. $\frac{[5]}{[7}$

A later experiment performed at SLAC showed that the EMC effect has the same qualitative behaviour for all nuclei, differing only in the value of the ratio at the minimum. ${ }^{8}$ It also showed that the EMC effect is independent of $Q^{2}$ for $2 \leq Q^{2} \leq 40(\mathrm{GeV} / \mathrm{c})^{2}$ and that the depth of the minimum at $x_{p} \approx 0.7$ grows with nuclear mass. The growth seemed to increase with the average nuclear density ${ }^{8}$ and this became a generally accepted feature of the EMC effect (see Ref. ${ }^{9}$ and references therein).

As theorists provided several different, simple, explanations of the effect (see discussion in section 2.2), an independent experimental test of these explanations was needed 10 This came from Fermi National Accelerator Lab in the form of DrellYan measurements. 11 These experiments compared $\mu^{+}-\mu^{-}$production from $q-\bar{q}$ annihilation in proton-proton and proton-nucleus collisions. In the kinematic range covered by the measurement, they observed that the nuclear to proton ratio was consistent with unity. As this experiment was sensitive mainly to the nuclear sea quarks, the result pointed to the EMC effect being due to a change in the valence quark distributions. 
It was not until 2009 that the simple nuclear density dependence of the EMC effect was challenged with new data ${ }^{12} \mathrm{~A}$ high precision measurement on light nuclei, including ${ }^{3} \mathrm{He},{ }^{4} \mathrm{He},{ }^{9} \mathrm{Be}$ and ${ }^{12} \mathrm{C}$, showed that the effect was not related to the average nuclear density. The most significant outlier was ${ }^{9} \mathrm{Be}$ which has a low average nuclear density, similar to that of ${ }^{3} \mathrm{He}$, and a large EMC effect, similar to that of ${ }^{4} \mathrm{He}$ and ${ }^{12} \mathrm{C}$ (see Fig. 2). This anomaly was consistent with variational Monte Carlo calculations which show that local, high density configurations occur in nuclei $\frac{13}{13}$ These calculations describe ${ }^{9} \mathrm{Be}$ as a collection of two alpha clusters and an orbiting neutron. In this picture, ${ }^{9} \mathrm{Be}$ has a low average density and a much higher local density similar to that of ${ }^{4} \mathrm{He}$. Thus, the phenomenological explanation of the EMC effect shifted, based on the new data, from an average density effect to a local density effect.

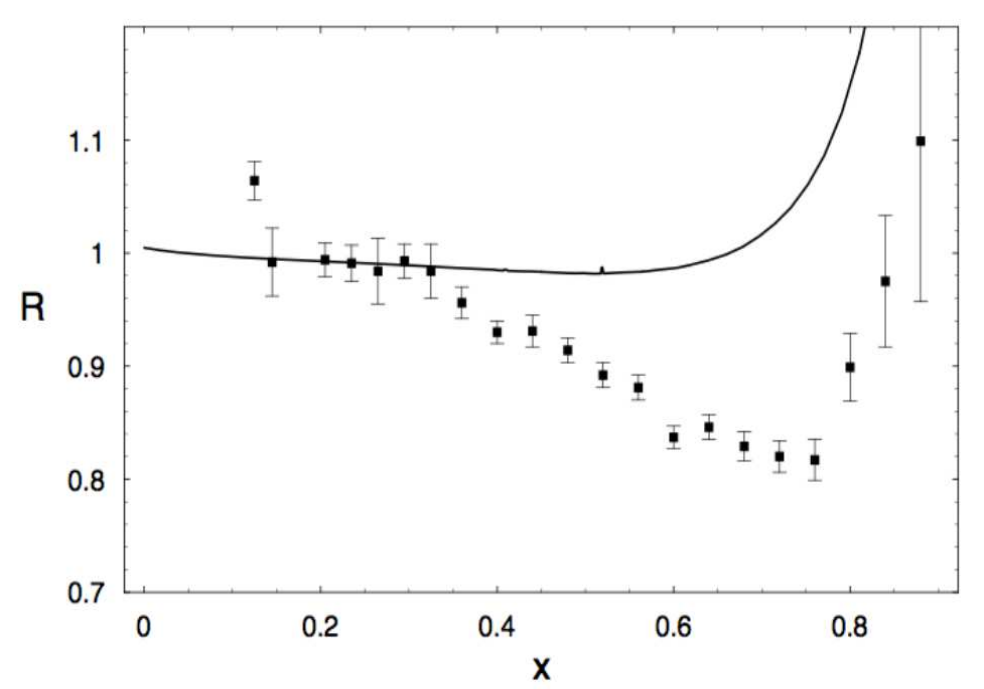

Fig. 1: Measurements of the DIS cross section ratio of gold relative to deuterium as a function of Bjorken- $\mathrm{x}_{p}$ from SLAC. The solid black line is the expected ratio taking into account only Fermi motion of nucleons in Gold. (Figure reprinted from.14 Copyright (2002) by the American Physical Society.)

\subsection{Theory Status}

In $\mathrm{QCD}$, the nucleon structure function, $F_{2}\left(x, Q^{2}\right)$, gives the weighted probability for finding a parton (quark) in the nucleon that carries a fraction $x$ of the total nucleon momentum. The different partons contribute with a weight equal to the square of their electric charge. The primary theory interpretation of the reduction 


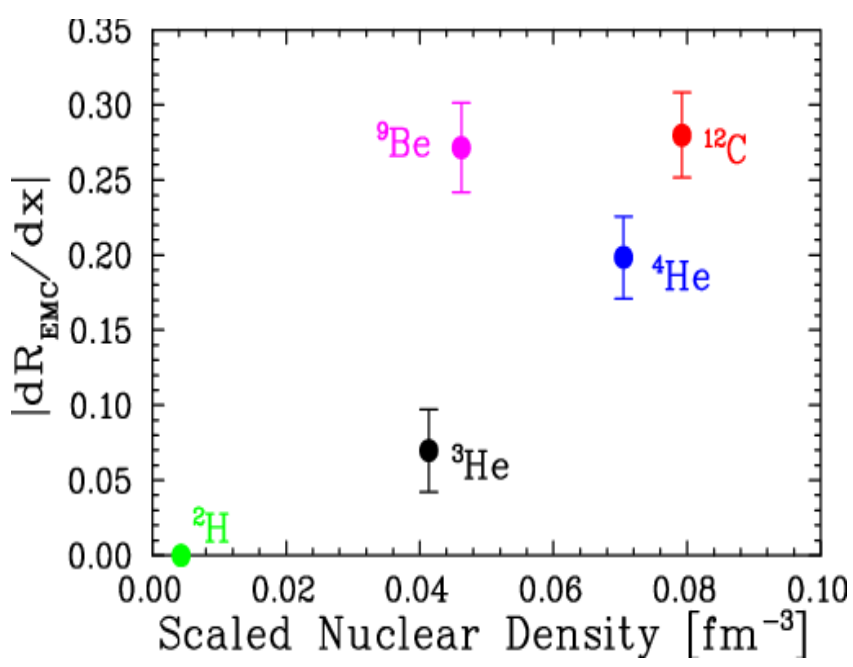

Fig. 2: The strength of the EMC effect, defined as the slope of the per nucleon DIS cross section ratio for $0.3 \leq x \leq 0.7$, shown as a function of the scaled nuclear density for light nuclei. (Figure reprinted from ${ }^{12}$ Copyright (2009) by the American Physical Society.)

of the nuclear structure function in the valence quark region was simple $19-15$ Quarks in nuclei carry less momentum than quarks in nucleons and, as the uncertainty principle implies, move throughout a larger confinement volume. This notion gave rise to a host of models: bound nucleons are larger than free ones; quarks in nuclei move in 6 quark or 9 quark or even $3 A$ quark bags. ${ }^{202}$ But more conventional explanations such as the influence of nuclear binding or enhancement of pion cloud effects were successful in reproducing some of the nuclear deep inelastic scattering data. $23+27$ And one could combine various different models. 10,28 This led to a plethora of models that reproduced the data, causing one of the present authors to write that EMC means Everyone's Model is Cool ${ }^{29}$ It is interesting to note that none of the earliest models were concerned with the role of two nucleon correlations, except as relating to six quark bags.

The initial excitement tapered off as nuclear deep inelastic scattering became more understood, the experimental data became more precise, and the need to include the effects of nuclear shadowing was acknowledged $\sqrt[30]{ }$ Indeed some of the more extreme models were ruled out by a failure to match well-known nuclear phenomenology. Moreover, inconsistency with the baryon and momentum sum rules led

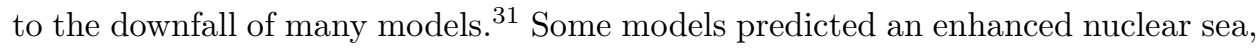
but others did not. As results from Drell-Yan measurements were published, none of the existing models survived the challenge of providing an accurate description of both the EMC and Drell-Yan data sets - a challenge that remains to this day.

It is now understood that conventional nuclear binding effects can account for 
O. Hen et al.

the EMC effect up to values of $x_{p} \approx 0.5$ or sc $\sqrt[14]{32}[35$ but fail at larger values. Therefore the effects of the nuclear modification of the nucleon structure function must be included. Currently viable models of nucleon modification include (a) the quark meson coupling model in which quarks in nucleons (either bags or eigenstates of the NJL model ${ }^{36}[37$ exchange mesons with quarks in other nucleons, (b) the chiral quark soliton model in which quarks in nucleons also exchange mesons with other nucleons, $\frac{38}{,}$ and (c) the suppression of point-like-configurations of the nucleon by the nuclear medium $\sqrt[39]{40}$ A successful phenomenology that includes the effects of shadowing, binding, pion enhancement and a medium-modification of the quark structure function can be fitted to the extant data. $\underline{41}$

A modern model which incorporates the influence of nucleon-nucleon correlations in a manner consistent with nuclear physics knowledge to describe both nuclear deep inelastic scattering and Drell-Yan data does not yet exist.

\subsection{EMC Data Analysis}

Following the EMC collaboration, other experiments measured the ratios of pernucleon DIS cross sections for nuclei and the deuteron at equal values of $Q^{2}$ and $x_{p}=Q^{2} / 2 m_{p} \omega$. In these kinematical conditions, the DIS cross section ratio for nuclei $A_{1}$ and $A_{2}$ is given by $\underline{\underline{42}}$

$$
\frac{\sigma_{A_{1}}}{\sigma_{A_{2}}}=\frac{F_{2}^{A_{1}}\left(x_{p}, Q^{2}\right)}{F_{2}^{A_{2}}\left(x_{p}, Q^{2}\right)} \cdot \frac{\left[1+2 \frac{1+\omega^{2} / Q^{2}}{R_{A_{1}}-1} \tan ^{2} \frac{\theta}{2}\right]}{\left[1+2 \frac{1+\omega^{2} / Q^{2}}{R_{A_{2}}-1} \tan ^{2} \frac{\theta}{2}\right]},
$$

where $\theta$ is the lepton scattering angle and $R_{A}=\sigma_{L}^{A} / \sigma_{T}^{A}$ is the ratio of the longitudinal to transverse cross section for nucleus A. Assuming $R_{A}$ independent of $A$, the cross section ratio of Eq. 2 is reduced to the the $F_{2}$ structure function ratio.

Recently, Frankfurt \& Strikman (FS) pointed out that the structure functions of nucleons bound in nuclei should be extracted in the reference frame of the nucleus. $\stackrel{34}{34}$ This is done by using the $x_{A}$ scaling variable, defined as:

$$
x_{A}=\frac{Q^{2}}{2 q \cdot P_{A} / A}=\frac{A Q^{2}}{2 \omega m_{A}}=x_{p} \cdot \frac{A m_{p}}{m_{A}}
$$

where $q$ and $P_{A}$ are the 4-momentum vectors of the virtual photon and target nucleus respectively, and $m_{A}$ is the mass of the target nucleus. Note that, for the same values of $Q^{2}$ and $\omega, x_{A}$ differs from $x_{p}$ by the ratio of the bound nucleon mass to the free mass. Therefore, a cross section measured at $Q^{2}$ and $\omega$ on nucleus $A$ will depend on the nucleon structure function evaluated at $x_{A}$ rather than at $x_{p}$.

This means that the standard EMC cross section ratio at the same $Q^{2}$ and $\omega$ (and hence the same $x_{p}$ ) is actually proportional to the nucleon structure function in nucleus $A$ evaluated at parton momentum fraction $x_{A}=A Q^{2} / 2 m_{A} \omega$ divided by the nucleon structure function in deuterium evaluated at parton momentum 
fraction $x_{d}=2 Q^{2} / 2 m_{d} \omega$. For symmetric nuclei this is:

$$
\frac{2}{A} \frac{\sigma_{D I S}^{A}\left(x_{p}, Q^{2}\right)}{\sigma_{D I S}^{d}\left(x_{p}, Q^{2}\right)}=\frac{F_{2}^{A}\left(x_{A}, Q^{2}\right)}{F_{2}^{d}\left(x_{d}, Q^{2}\right)}
$$

where $\frac{\sigma_{D I S}^{A}}{\sigma_{D I S}^{d}}$ is the DIS cross section ratio measured at the same $\left(Q^{2}, \omega, x_{p}\right)$, and $\frac{F_{2}^{A}\left(x_{A}, Q^{2}\right)}{F_{2}^{d}\left(x_{d}, Q^{2}\right)}$ is the ratio of structure functions at the same $\left(Q^{2}, \omega\right)$ but different $x$. Since we want to compare the structure functions at the same parton momentum fractions, we want to correct this using

$$
\frac{F_{2}^{A}\left(x_{A}, Q^{2}\right)}{F_{2}^{d}\left(x_{d}, Q^{2}\right)}=\frac{F_{2}^{A}\left(x_{A}, Q^{2}\right)}{F_{2}^{d}\left(x_{A}, Q^{2}\right)} \cdot \frac{F_{2}^{d}\left(x_{A}, Q^{2}\right)}{F_{2}^{d}\left(x_{d}, Q^{2}\right)}
$$

and

$$
\frac{F_{2}^{A}\left(x_{A}, Q^{2}\right)}{F_{2}^{d}\left(x_{A}, Q^{2}\right)}=\frac{\sigma_{D I S}^{A}\left(x_{p}, Q^{2}\right)}{\sigma_{D I S}^{d}\left(x_{p}, Q^{2}\right)} \cdot \frac{F_{2}^{d}\left(x_{d}, Q^{2}\right)}{F_{2}^{d}\left(x_{A}, Q^{2}\right)}
$$

where $\frac{F_{2}^{A}\left(x_{A}, Q^{2}\right)}{F_{2}^{d}\left(x_{A}, Q^{2}\right)}$ is the ratio of structure functions in the different nuclei evaluated at the same parton momentum fraction (i.e., the quantity we wish to extract), and $\frac{F_{2}^{d}\left(x_{A}, Q^{2}\right)}{F_{2}^{d}\left(x_{d}, Q^{2}\right)}$ is a correction factor. This correction factor can be evaluated using well known parameterizations of the deuteron structure function. $43[44$

Fig. 3 shows the effect of the correction factor of Eq. 6 on the measured DIS cross section ratio for ${ }^{12} \mathrm{C}$ relative to deuterium from recent Jefferson Lab measurements. As can be seen, the $x_{A}$ correction reduces the size of the EMC effect (i.e., its slope). It replaces part of the model-dependent binding energy corrections with a systematic, transparent, and model-independent correction.

For asymmetric nuclei $(N \neq Z)$, following Aubert et al. and Bodek et al., 1,3 an additional isoscalar correction factor $\left(R_{I S O}\right)$ is applied to the measured cross section ratio, making it related to a hypothetical nucleus with equal number of protons and neutrons $(N=Z=A / 2)$ :

$$
\frac{\sigma_{D I S}^{A}\left(x_{A}, Q^{2}\right)_{I S O}}{\sigma_{D I S}^{d}\left(x_{A}, Q^{2}\right)_{I S O}}=\frac{\sigma_{D I S}^{A}\left(x_{A}, Q^{2}\right)}{\sigma_{D I S}^{d}\left(x_{A}, Q^{2}\right)} \cdot R_{I S O}\left(x_{A}\right),
$$

with $R_{I S O}\left(x_{A}\right)$ defined as:

$$
R_{I S O}\left(x_{A}\right)=\frac{A}{2} \frac{F_{2}^{p}\left(x_{A}, Q^{2}\right)+F_{2}^{n}\left(x_{A}, Q^{2}\right)}{Z \cdot F_{2}^{p}\left(x_{A}, Q^{2}\right)+N \cdot F_{2}^{n}\left(x_{A}, Q^{2}\right)}=\frac{A}{2} \frac{1+R_{n p}\left(x_{A}, Q^{2}\right)}{Z+N \cdot R_{n p}\left(x_{A}, Q^{2}\right)},
$$

where $F_{2}^{p}\left(x_{A}, Q^{2}\right)$ and $F_{2}^{n}\left(x_{A}, Q^{2}\right)$ are the free proton and neutron structure functions, and $R_{n p}\left(x_{A}, Q^{2}\right)=F_{2}^{n}\left(x_{A}, Q^{2}\right) / F_{2}^{p}\left(x_{A}, Q^{2}\right)$. The free neutron structure function used in this correction is usually extracted from world data on DIS scattering off deuterium and the proton, corrected for the Fermi motion of protons and neutrons in deuterium (i.e., smearing effect), see $\frac{\sqrt{45}[46}{46}$ for details. 
Using the isoscalar correction for asymmetric nuclei and the description of the measured cross section ratios in terms of $F_{2}^{A}$ and $F_{2}^{d}$ (Eq. 5 ), we extract the structure function ratio of nucleons bound in nuclei relative to deuterium as:

$$
\begin{aligned}
\frac{F_{2}^{A}\left(x_{A}, Q^{2}\right)}{F_{2}^{d}\left(x_{A}, Q^{2}\right)} & =\frac{2}{A} \frac{\sigma_{D I S}^{A}\left(x_{p}, Q^{2}\right)}{\sigma_{D I S}^{d}\left(x_{p}, Q^{2}\right)} \cdot \frac{F_{2}^{d}\left(x_{d}, Q^{2}\right)}{F_{2}^{d}\left(x_{A}, Q^{2}\right)} \cdot R_{I S O}\left(x_{A}\right)= \\
& =\frac{2}{A} \frac{\sigma_{D I S}^{A}\left(x_{p}, Q^{2}\right)_{I S O}}{\sigma_{D I S}^{d}\left(x_{p}, Q^{2}\right)_{I S O}} \cdot \frac{F_{2}^{d}\left(x_{d}, Q^{2}\right)}{F_{2}^{d}\left(x_{A}, Q^{2}\right)} \cdot \frac{R_{I S O}\left(x_{A}\right)}{R_{I S O}\left(x_{p}\right)}
\end{aligned}
$$

Appendix A presents the EMC ratios, extracted as a function of $x_{A}$ for $0.3 \lesssim$ $x_{A} \lesssim 0.7$, for all nuclei measured at SLAC ${ }^{12}$ and JLab ${ }^{[8}$ The isoscalar correction applied is identical for both data sets, making them more consistent.

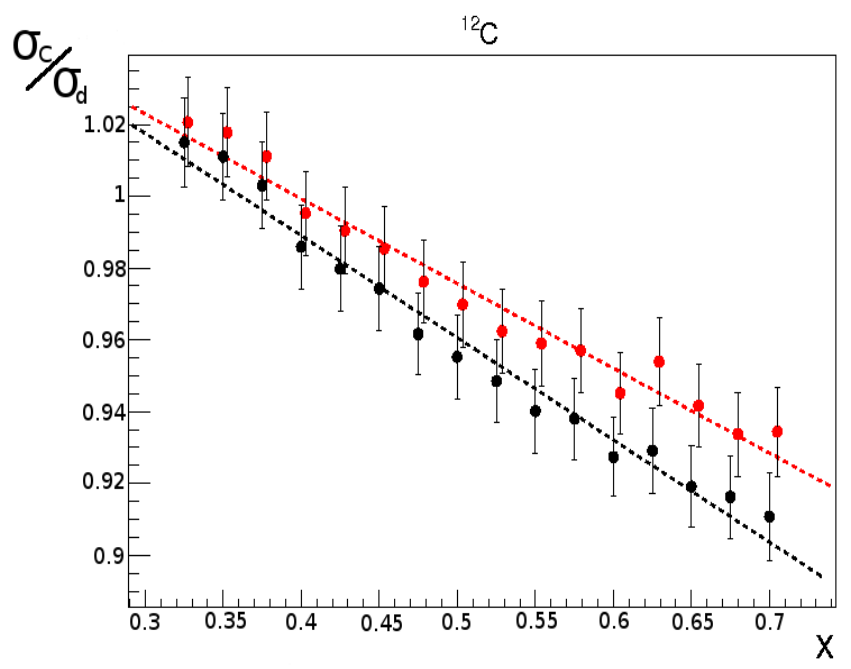

Fig. 3: Example of the effect of the $x_{A}$ correction to the data. The black points are the original Seely data plotted as a function of $x_{p}$. The red points are the corrected data, plotted as a function of $x_{A}$. Dashed lines are linear fits to the two data sets. The difference between the slope of the two fits is about $20 \%$.

\section{High momentum nucleons in nuclei}

This section describes nucleon-nucleon (or "two-nucleon") correlations. A correlated two-nucleon pair is one where the two-nucleon density is significantly different from the product of two single-nucleon densities. Both tensor and central forces can produce short-range correlations. 


\subsection{Theoretical Need for High Momentum Nucleons in Nuclei}

The strong interactions between nucleons in nuclei are dominated by two and three nucleon terms. Therefore the fact that nucleons in nuclei are correlated is selfevident. There is no fundamental one-body potential in the nucleus, unlike the onebody Coulomb potential in atomic physics. The fundamental question of nuclear physics was: how does the very successful shell model of the nucleus emerge in spite of the strong short-ranged interactions between nucleons? An answer was provided early on by Brueckner \& Goldstone, see the review by Bethe .47 The strong twonucleon interactions encoded by the potential $V$, constructed to reproduce experimentally measured scattering observables and believed to include strong repulsion at short distance and attraction at longer ranges, are summed to form the $T$ matrix of scattering theory and the $G$-matrix for bound states. The operator $G$ is obtained from $T$ by modifying the propagator to include the effects of the Pauli principle and to use the appropriate self-consistent (single) nucleon energies. The $G$ matrix is considerably weaker than $V$, and can therefore be used in perturbation theory. One forms the nuclear mean field $U$ throughout the Hartree-Fock method employing the $G$-matrix, and the first approximation to the wave function is the anti-symmetrized product of single particle wave functions engendered by $U$. However, the complete nuclear wave function is obtained in a perturbative hole-line expansion that includes two-particle - two-hole excitations and other excitations which incorporate correlations. Later work formulated a relativistic version of Brueckner theory in which the Dirac equation replaces the Schroedinger equation. 48 There is also a light front version. 50 [51

The Brueckner theory approach described above presumes that the two-nucleon potential contains strong short-distance repulsion. Early attempts to construct soft potentials lacking the strong repulsion that also reproduce scattering data did not succeed in obtaining interactions that could be used perturbatively in the nuclear bound state problem. $\cdot 77$ In modern times, the use of effective field theory provides a low-energy version of $\mathrm{QCD}$, guided by chiral symmetry, in which one obtains the potential as an expansion in powers of $\left(Q / \Lambda_{\chi}\right)$ where $Q$ is a generic external momentum (nucleon momentum) and $\Lambda_{\chi}$ is the chiral symmetry breaking scale of about $1 \mathrm{GeV}$. See the review ${ }^{[52} \mathrm{In}$ such theories the short distance interaction can be treated as a contact interaction, modified by the inclusion of a cut-off, and the longer ranged interactions are accounted for by one and two pion exchange interactions. The softness (involving low momentum) or hardness (involving higher momentum) of the potential is determined by the value of the cutoff. For sufficiently soft potentials nuclear matter can be treated using perturbation theory in terms of the two and three nucleon chiral interactions. Nevertheless, two-nucleon correlations occur, primarily as a result of the second iteration of the one pion exchange potential.

Another approach uses renormalization group methods to generate a soft $N N$ potential from a hard interaction either by integrating out high momentum compo- 
nents (in the case of $V_{\text {low }-K}$ ), or by using the similarity renormalization group $\frac{53}{53}$ Then one obtains a potential that is mainly restricted to small values of momentum. This potential is perturbative in the sense that the Born series for scattering converges and perturbation theory can be applied to the nuclear bound state problem. However, once again the second-order term in the potential generates correlations.

The renormalization group can be used to eliminate matrix elements of the nucleon-nucleon potential connecting low and high relative momentum states. Such a procedure simplifies the computations of nuclear binding energies and spectra, and would also lead to wave functions without high-momentum components and truly short ranged-correlations. However, it would be necessary to consistently transform all other operators. For high momentum transfer reactions, the renormalization group changes a known simple probe, described by a single-nucleon operator into a complicated probe describable by unknown (in practice) $A$-nucleon operators. This prevents the analysis of any high momentum transfer experiment.

To summarize, there are two basic approaches to fundamental nuclear structure - perturbation in the $G$ matrix or perturbation in the potential. In either case there will be two-nucleon correlations. Theoretically, the key remaining question concerns the quantity and range of the correlations.

\subsection{SRC Measurements}

Experimentalists at electron scattering facilities such as SACLAY and NIKHEF observed the need for high momentum components in nuclei, not from direct observation, but rather from a dramatic lack of cross section in $A\left(e, e^{\prime} p\right) A-1$ valence shell knock-out experiments where the independent particle models overestimated the measured cross sections. ${ }^{54}$ Since the shell model accurately predicts energy levels and spins, this reduced the range of possible explanations. The most straightforward explanation was that the "missing nucleons" were in nucleon-nucleon correlations. When the electron scattered from a nucleon in a correlated pair, its partner was also ejected from the nucleus. This shifted strength from excitation energies typical of valence states to much higher excitation energies.

Many experiments were done at these facilities to probe for more direct evidence of correlations; but as history would show, the necessary kinematic requirements, $x_{B}>1$ and $Q^{2}>1(\mathrm{GeV} / \mathrm{c})^{2}$, were practically inaccessible. Thus most of the early experiments ended up being studies of reaction mechanisms such as meson-exchange currents and final-state interactions.

With the availability of continuous, high intensity, high momentum proton and electron beams, identifying SRCs in Quasi-Elastic (QE) scattering off nuclei became feasible. In this section we review results from measurements of inclusive $\mathrm{QE}\left(e, e^{\prime}\right)$ cross section ratios and exclusive, triple-coincidence, $\left(e, e^{\prime} p N\right)$ and $(p, 2 p n)$, large momentum transfer (hard), reactions performed at the Stanford Linear Accelerator Center (SLAC), Brookhaven National Lab (BNL) and more recently at Jefferson Lab (JLab). 


\subsubsection{Inclusive SRC Measurements}

In inclusive scattering of unpolarized particles from an unpolarized target, there are only two independent kinematical variables. In the case of inclusive QE electron scattering these are normally chosen to be $Q^{2}$ and $x_{p}$. In the Plain Wave Impulse Approximation (PWIA) it is assumed that the virtual photon is fully absorbed on a single nucleon, which leaves the nucleus without rescattering, leaving the remaining $A-1$ nuclear system unperturbed. Energy and momentum conservation for such a reaction define a minimum value for the component of the initial momentum of the scattered nucleon in the direction of the virtual photon as a function of $Q^{2}$ and

$x_{p} .55$ At $x_{p}=1$, for all $Q^{2}$ values, the minimum value of this momentum component equals zero. As one increases or decreases $x_{p}$ at fixed $Q^{2}$, its value increases. At moderate values of $Q^{2}\left(\sim 2-4 \mathrm{GeV} / \mathrm{c}^{2}\right)$ and $x_{p} \geq 1.4-1.5(\leq \sim 0.6)$ this minimum value is larger than the Fermi momentum $\left(k_{F}\right)$ of the nucleus, and the reaction is dominated by scattering from high momentum $\left(\geq k_{F}\right)$ nucleons in the nucleus. At these $Q^{2}$ values and $x_{p}<1$, the virtual photon carries a large amount of energy compared to its momentum and the reaction, while sensitive to high momentum nucleons, has large inelastic contributions from $\Delta$ production and meson exchange currents (MEC). On the other hand, for the same $Q^{2}$ values and $x_{p}>1$, the virtual photon transfers a small amount of energy compared to its momentum, inelastic processes are suppressed, and the reaction is more directly sensitive to the nature of the high momentum tail of the nuclear wave function.56 57 In both cases, large values of $Q^{2}$ suppress meson exchange current (MEC) contributions $\frac{58][59}{[59}$

Inclusive electron scattering cross section ratios for nucleus $A$ relative to deuterium and to ${ }^{3} \mathrm{He}$ were measured at SLAC and later at Hall-B and Hall-C of JLab $\sqrt[55[60] 62]]{6 i g}$. 4 4 shows the $x_{p}$ dependence of the per nucleon cross section ratio of nuclei relative to ${ }^{3} \mathrm{He}$ measured at Hall-B. As can be seen, for $x_{p}$ values which correspond to scattering off high momentum $\left(\geq k_{F}\right)$ nucleons in the nucleus (i.e., $1.5 \leq x_{p} \leq 2$ and $x_{p} \geq 2.25$ ) the cross section ratio scales (i.e., does not depend on $x_{p}$ ). The contribution of Final State Interactions (FSI) to the measured cross sections are expected to rapidly decrease as a function of $Q^{2}$. Calculations of FSI in inclusive scattering at large $Q^{2}$ and $x_{p} \geq 1$ show they are largely confined to within the nucleons of the initial-state SRC pair.56] The contribution of FSI of this kind will cancel in the cross section ratio of two nuclei. This is supported by the small observed $Q^{2}$ dependence of the cross section scaling plateau. This scaling reflects the scaling of the high momentum tail of the nuclear wave function and is usually interpreted using the Short-Range-Correlation (SRC) model. ${ }^{60 \mid 63}$ The latter states that the high momentum tail of the nuclear wave function is dominated by correlated, multi-nucleon, configurations. Due to their strong interaction at short distances, the structure of these configurations is independent of the surrounding nuclear environment, resulting in the same shape of the high momentum tail in all nuclei (i.e., scaling). Different nuclei have different amounts of short range correlated (SRC) clusters. In this model, the observed scaling of the per-nucleon cross 
September EMC'reviewMar15b

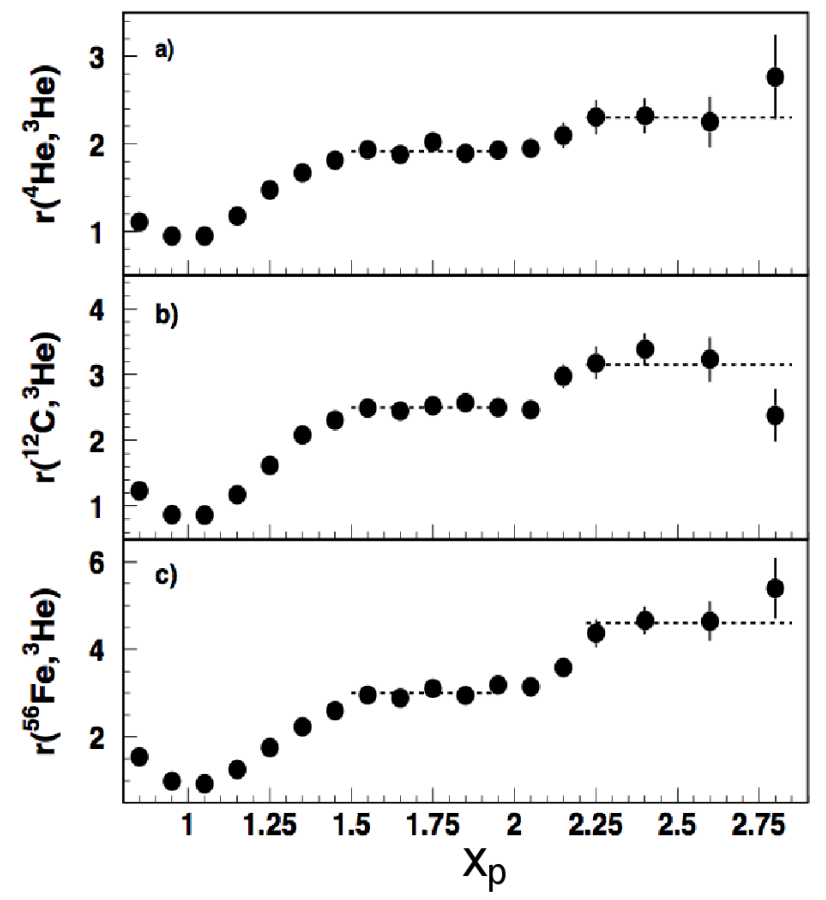

Fig. 4: Per nucleon QE inclusive $\left(e, e^{\prime}\right)$ scattering cross section ratios for nuclei relative to ${ }^{3} \mathrm{He}$ plotted as a function of $x_{p}$. Two plateaus are observed for $1.5 \leq$ $x_{p} \leq 2$ and $x_{p} \geq 2.25$. The magnitude of these plateaus are labeled as $a_{2}$ and $a_{3}$, respectively. In the SRC model of the high momentum tail of the nuclear wave function, they are taken as a measure of the relative amount of $2 \mathrm{~N}$ and $3 \mathrm{~N}$ SRC pairs in the measured nuclei. See text for more details. (Figure reprinted from. 61 Copyright (2006) by the American Physical Society.)

section ratios for $1.5 \leq x_{p} \leq 2$ and $x_{p} \geq 2.25$ are indicative of scattering off twonucleon $(2 \mathrm{~N})$ and three-nucleon $(3 \mathrm{~N}) \mathrm{SRC}$ configurations, respectively. The scaling factors, noted as $a_{2}$ and $a_{3}$ are then a measure of the relative amount of $2 \mathrm{~N}$ and $3 \mathrm{~N}$ SRC, respectively, in the measured nuclei.

\subsubsection{Exclusive (p,2pn) and (e,e'pN) Measurements}

Inclusive measurements alone do not prove that high momentum nucleons are a result of initial-state short-range correlated pairs. To study the contribution of $2 \mathrm{~N}$ SRC pairs to the high momentum tail of the nuclear wave function exclusive twonucleon-knockout experiments were done. The concept behind such experiments is that, in the Plain-Wave-Impulse-Approximation (PWIA), in the absence of FSI, when a nucleon that is part of a $2 \mathrm{~N}-\mathrm{SRC}$ pair is knocked out the nucleus, in order 
to conserve momentum its correlated partner nucleon has to recoil with momentum that is equal in size and opposite in direction to the initial momentum of the knocked-out nucleon. This back-to-back correlation between the initial momentum of the knocked-out nucleon and the momentum of the recoil nucleon, both above the Fermi sea level $k_{F}$, is a clear signature for scattering off a $2 \mathrm{~N}-\mathrm{SRC}$ configuration. Due to the center-of-mass (c.m.) motion of the $2 \mathrm{~N}-\mathrm{SRC}$ pair with respect to the residual $A-2$ nuclear system, this correlation will not be exactly back-to-back. The measured angular correlation can be used to extract the c.m. momentum distribution of the pair. If the $2 \mathrm{~N}-\mathrm{SRC}$ model is correct, the nucleons in the pair will have large relative momentum $\left(\geq k_{F}\right)$ and small c.m. momentum $\left(\leq k_{F}\right)$.

Two nucleon knockout experiments, measuring the ${ }^{12} \mathrm{C}(p, 2 p n)$ and ${ }^{12} \mathrm{C}\left(e, e^{\prime} p N\right)$ reactions, were done at BNL and JLab, respectively ${ }^{64} 67$ These experiments scattered protons and electrons off high initial momentum $\left(300 \leq P_{\text {initial }} \leq 600 \mathrm{MeV} / \mathrm{c}\right)$ protons in ${ }^{12} \mathrm{C}$ and looked for the emission of a correlated recoil nucleon. In the absence of FSI, the initial momentum of the struck nucleon equals the missing momentum of the ${ }^{12} C\left(e, e^{\prime} p\right)$ and ${ }^{12} C(p, 2 p)$ reactions. FSI will make this relationship more approximate. For simplicity, we will ignore FSI The JLab measurement was sensitive to both proton and neutron recoils but the BNL measurement was only sensitive to recoiling neutrons. These experiments were performed at large momentum transfer $\left(Q^{2} \approx 2 \mathrm{GeV} / \mathrm{c}^{2}\right)$ where competing effects such a Meson-ExchangeCurrents (MEC) and Isobar Contributions (IC) are suppressed and FSI are mainly confined to be between the nucleons of the pair. The main results of these experiments are shown in Figs. 5 and 6 , Fig. 5 shows the distribution of the cosine of the opening angle between the initial momentum of the knocked-out proton and the recoil nucleon. The c.m. motion of the pairs in both cases was found to be consistent with a gaussian in each direction, with $\sigma=143 \pm 17$ (BNL) and $\sigma=136 \pm 20$ (JLab). The BNL results show a clear threshold around the fermi momentum where recoiling neutrons above this momentum show a clear angular correlation, and those below it do not. Fig. [6 shows the ratio of single nucleon knockout events to two nucleon knockout events, corrected for finite acceptance effects, as a function of the initial momentum of the knocked-out proton. As can be seen, within statistical uncertainties, all single proton knockout events were accompanied by the emission of a recoil nucleon. The ratio of proton recoil to neutron recoil was found to be approximately 1:20 ${ }^{67}$ This is a clear evidence of the importance of the tensor part of the nucleon-nucleon interaction at these momentum scales $\underline{68}[\underline{69}$

The effect of these measurements on our understanding of the short distance nuclear structure is illustrated by the pie chart shown in Fig. 6. From the inclusive cross section ratio measurements and from $A\left(e, e^{\prime} p\right)$ measurements we know that in medium and heavy nuclei (i.e., $A \geq 12) \sim 75-80 \%$ of the nucleons are 'Mean-Field' nucleons, whereas $\sim 20-25 \%$ have momentum greater than the Fermi momentum of the nucleus. Combined with results from exclusive two-nucleon knockout measurements we know that these high momentum nucleons are dominated by $2 N$-SRC pairs, which are in turn dominated by neutron-proton pairs. 
September EMC'reviewMar15b
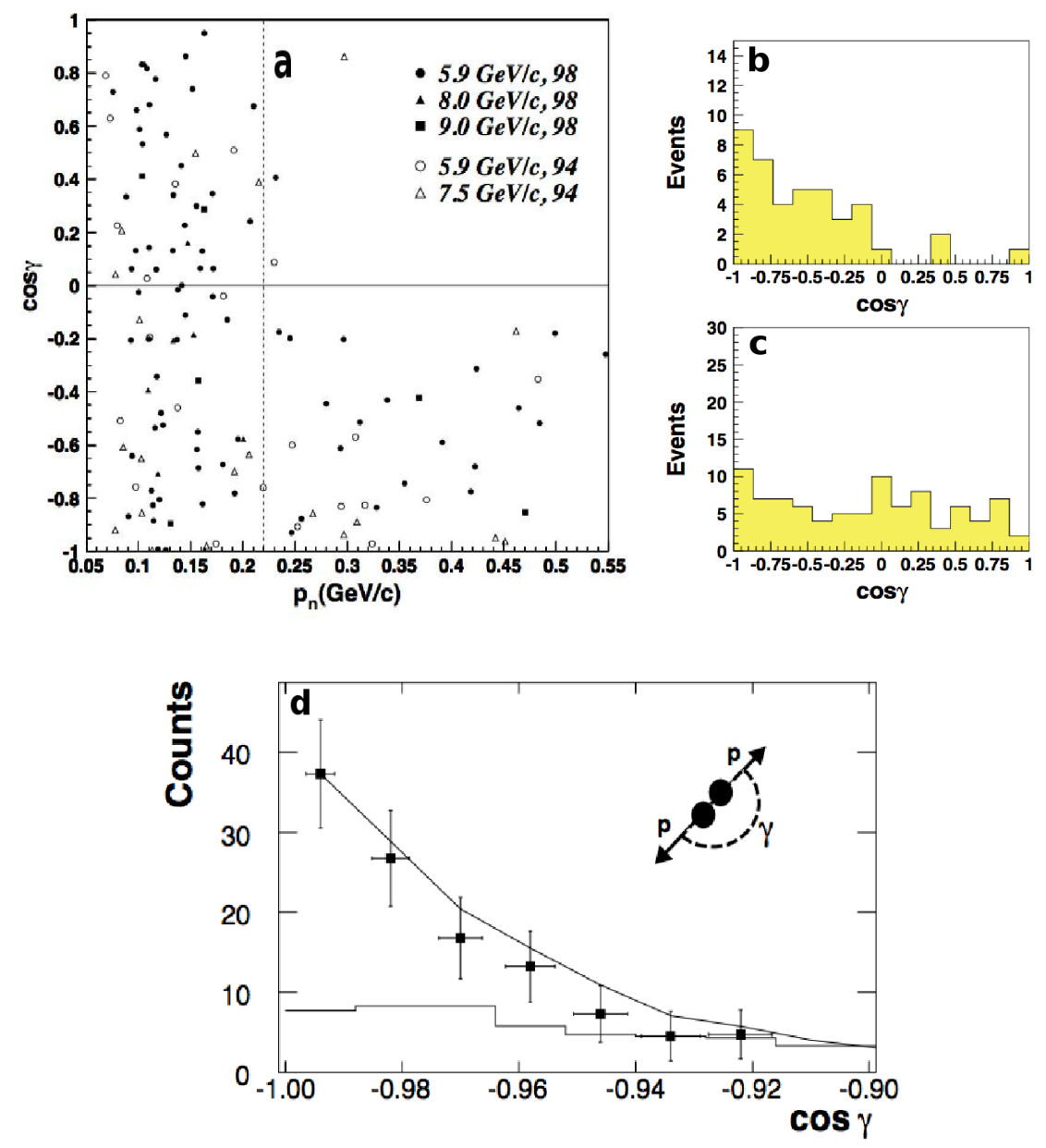

Fig. 5: Distributions of the relative angle $(\gamma)$ between the reconstructed initial momentum of the knockout proton and the recoil nucleon. Top: Results for proton induced proton-neutron pair knockout (i.e. ${ }^{12} \mathrm{C}(p, 2 p n)$ ) measurements from BNL, shown and a function of: (a) the momentum of the recoil neutron, (b) for events with recoiling neutron with momentum greater then the Fermi momentum, and (c) for events with recoiling neutron with momentum lower then the Fermi momentum. These results show a clear transition from an isotropic distribution to a back-to-back correlated distribution as the recoil neutron momentum reaches the Fermi momentum of ${ }^{12} \mathrm{C}$. Bottom: Results for electron induced proton-proton pair knockout (i.e. $\left.{ }^{12} \mathrm{C}\left(e, e^{\prime} p p\right)\right)$ measurements from JLab, shown for events in which the initial momentum of the knockout proton, $|\vec{p}-\vec{q}|$, equals $\sim 500 \mathrm{MeV} / \mathrm{c}$. (Figures reprinted from ${ }^{64}$ Copyright $(2003,2006,2007)$ by the American Physical Society.) 

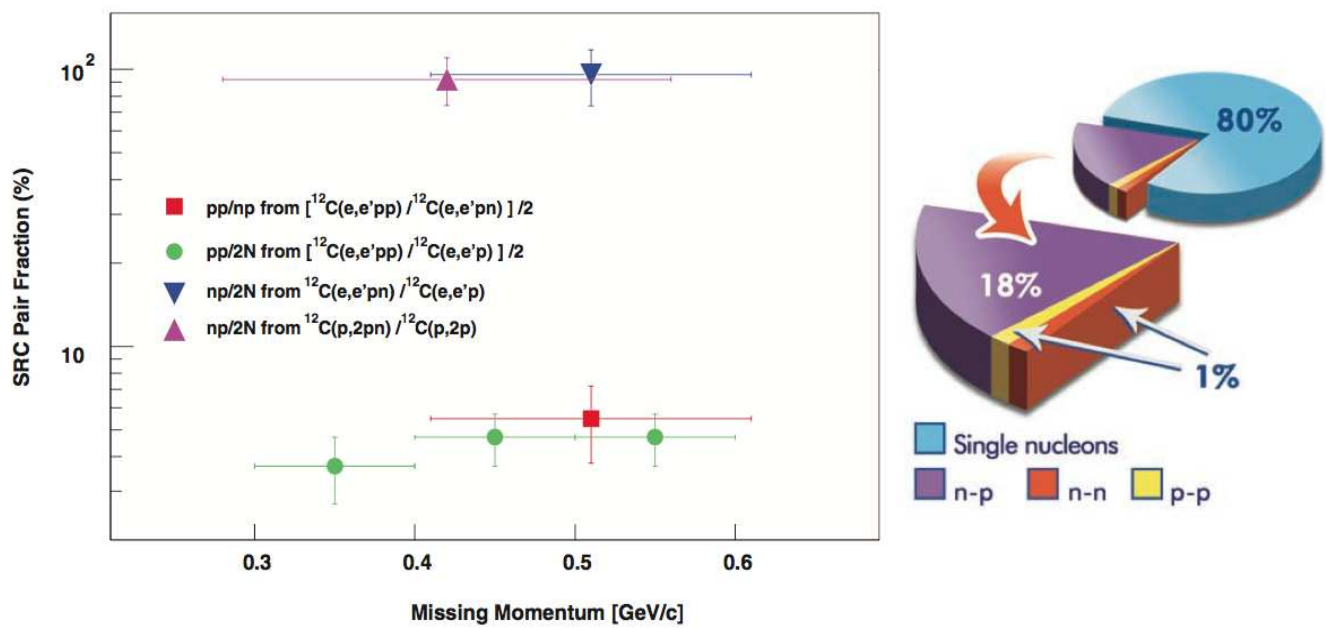

Fig. 6: The ratio of ${ }^{12} \mathrm{C}\left(e, e^{\prime} p N\right)$ double knockout events to ${ }^{12} \mathrm{C}\left(e, e^{\prime} p\right)$ single knockout events, shown as a function of the reconstructed initial (missing) momentum of the knocked-out proton from the ${ }^{12} \mathrm{C}\left(e, e^{\prime} p\right)$ reaction. Triangles and circles mark ${ }^{12} \mathrm{C}\left(e, e^{\prime} p n\right)$ and ${ }^{12} \mathrm{C}\left(e, e^{\prime} p p\right)$ events, respectively. The square shows the ${ }^{12} \mathrm{C}\left(e, e^{\prime} p p\right) /{ }^{12} \mathrm{C}\left(e, e^{\prime} p n\right)$ ratio. A clear dominance of ${ }^{12} \mathrm{C}\left(e, e^{\prime} p n\right)$ events is observed, evidence of the tensor nature of the nucleon-nucleon interaction in the measured momentum range. The pie chart on the right illustrates our understanding of the structure of ${ }^{12} \mathrm{C}$, composed of $80 \%$ mean-field nucleons and $20 \%$ SRC pairs, where the latter is composed of $\sim 90 \% n p$-SRC pairs and $5 \% p p$ and $n n$ SRC pairs each. (Figure reprinted with permission from American Association for Advancement in Science! 67

\section{Short Range Correlations and the EMC Effect}

\subsection{The EMC-SRC Correlation}

Analysis of world data on inclusive DIS and QE scattering cross section ratios showed that the magnitude of the EMC effect in nucleus $A$ is linearly related to the probability that a nucleon in that nucleus is part of a $2 N$-SRC pair, see Fig. 7$]^{70}$ Here we used the $x_{A}$ corrected EMC data-base shown in Appendix A and defined the magnitude of the EMC effect, following Ref., 12 as the slope of the ratio of the per-nucleon DIS cross section of nucleus $A$ relative to deuterium, $d R_{\mathrm{EMC}} / d x$, in the region $0.35 \leq x_{A} \leq 0.7$. The probability that a nucleon belongs to an SRC pair is characterized by the SRC scale factor, $a_{2}(A / d)$, the ratio in the plateau region $\left(Q^{2} \geq 1.5(\mathrm{GeV} / c)^{2}\right.$ and $\left.x_{p} \geq 1.5\right)$ of the per-nucleon $\mathrm{QE}\left(e, e^{\prime}\right)$ cross sections for nucleus $A$ and deuterium.

The EMC effect correlates imperfectly with other $A$-dependent quantities ( $\sec ^{12}[2]$ and references therein). In general, nuclei with $A \geq 4$ fall on one straight 
September EMC'reviewMar15b

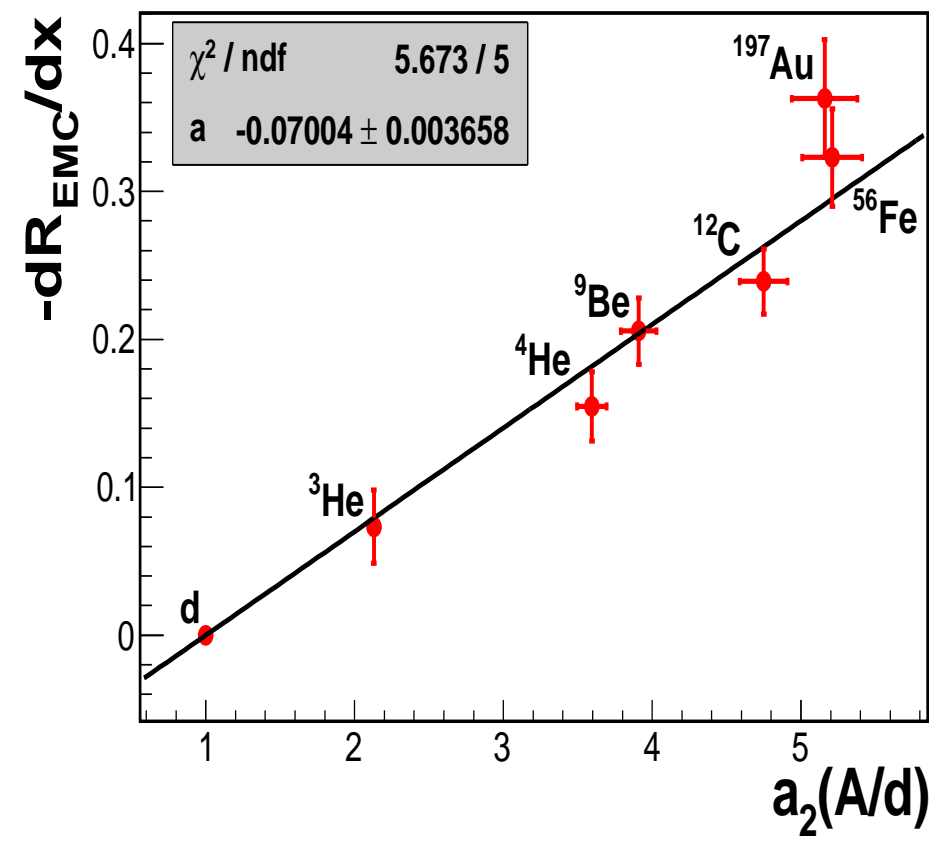

Fig. 7: The slope of the EMC effect $\left(R_{\mathrm{EMC}}\right.$, ratio of nuclear to deuteron cross section) for $0.35 \leq x_{A} \leq 0.7$ plotted vs. $a_{2}(A / d)$, the SRC scale factor (the relative probability that a nucleon belongs to an SRC $N N$ pair) for a variety of nuclei. ${ }^{71}$ The fit parameter, $a=-0.084 \pm 0.004$ is the intercept of the line constrained to pass through the deuteron (and is therefore also the negative of the slope of that line).

line but deuterium and ${ }^{3} \mathrm{He}$ do not. This is true when the EMC effect is plotted versus $A, A^{-1 / 3}$, or the average nuclear separation energy. When plotting the EMC effect versus average nuclear density, ${ }^{9} \mathrm{Be}$ is a clear outlier. This indicates that the excellent correlation with the SRC scale factor is not just a trivial byproduct of their mutual $A$ dependence.

The correlation between the EMC effect and the SRC scale factor is robust. ${ }^{71}$ It applies to both new SRC data sets of Egiyan et al., ${ }^{61}$ and Fomin et al. ${ }^{62}$ The quality of the correlation also does not depend on the corrections applied to the SRC data. These corrections include isoscalar cross section corrections, center-of-mass motion corrections and isoscalar pair-counting corrections. The isoscalar correction to the SRC scale factors accounts for the different elementary electron-neutron and electron-proton cross sections. This has a negligible effect on the fit quality and the extracted fit parameter. Fomin et al. did not apply this correction, arguing that short range correlations are dominated by $n p$ pairs. Fomin et al. argued that the 
SRC scale factors measured the relative probability of finding a high-momentum nucleon in nucleus $A$ relative to deuterium and that these scale factors needed to be corrected for the center-of-mass $(\mathrm{cm})$ motion of the pair in order to determine the relative probability that a nucleon in nucleus $A$ belongs to an SRC pair. As shown in both ${ }^{71}$ and ${ }^{72}$ including the pair c.m. motion correction improves the EMC-SRC correlation only slightly.

This EMC-SRC correlation gives new insight into the origin of the EMC effect. Many different explanations of the EMC effect have been proposed since 1983. After accounting for the standard nuclear effects of binding energy and fermi motion, explanations for the EMC effect fall into two general categories, those that require modifications of mean-field nucleons and those that require modifications of high-momentum nucleons. The linear correlation between the strength of the EMC effect and the SRC scale factors indicates that possible modifications of nucleon structure occurs in nucleons belonging to SRC pairs. This implies that the EMC effect, like short range correlations, is a short-distance, high virtuality, and high density phenomenon.

Additionally, one can use the EMC-SRC correlation as a phenomenological tool to constrain the deuteron IMC effecta, and thus extract the free neutron structure function. Following Weinstein et al., ${ }^{\sqrt{70}}$ we can extrapolate the linear fit to the EMCSRC correlation to the limit of $a_{2}(A / d) \rightarrow 0$. If the EMC effect and the SRC scale factor both stem from the same cause, then both will vanish at the same point. The value $a_{2}(A / d) \rightarrow 0$ is the limit of free nucleons with no SRC. The extrapolation to the $y$-axis gives $d R_{\mathrm{EMC}} / d x=-0.070 \pm 0.004$. Since the EMC effect is linear for $0.3 \leq x_{A} \leq 0.7$ for all nuclei with $A>2$, we assume that the EMC effect is also linear in this region for the free proton plus neutron. This gives the EMC effect for the free proton plus neutron:

$$
\frac{\sigma_{d}}{\sigma_{p}+\sigma_{n}}=1-a\left(x_{p}-b\right) \quad \text { for } 0.3 \leq x_{p} \leq 0.7,
$$

where $\sigma_{d}$ and $\sigma_{p}$ are the measured DIS cross sections for the deuteron and free proton, $\sigma_{n}$ is the free neutron DIS cross section that we want to extract, $a=$ $\left|d R_{\mathrm{EMC}}\right|=0.070 \pm 0.004$ and $b=0.34 \pm 0.02$ is the average value of $x_{p}$ where the EMC ratio is unityb. This implies that $\sigma_{d} /\left(\sigma_{p}+\sigma_{n}\right)$ decreases linearly from 1 to 0.97 as $x_{p}$ increases from 0.3 to 0.7 . We can then use this relationship to extract the free neutron cross section in this $x_{p}$ range. Incorporating this free neutron DIS cross section into the global QCD analysis, ${ }^{73}$ one can better constrain the $d / u$ ratio at $x_{p} \rightarrow 1$ to be equal to $0.23 \pm 0.09$ at the $90 \%$ confidence level ${ }^{74}$

The uncertainty quoted above is the uncertainty due to the data and the fit.

a The deuteron In Medium Correction (IMC) effect was first introduced in Ref ${ }^{70}$ and refers to the difference between the DIS cross section for the deuteron and the sum of the cross sections for a free proton and neutron.

${ }^{\mathrm{b}}$ While the $x_{A}$ correction does not change much the slope of the EMC-SRC correlation, the $b$ parameter extracted here, while consistent within errors, is larger than that reported in Ref. 70 
It does not include the uncertainty of corrections to the data. As stated above, if we include the correction for the $\mathrm{cm}$ motion of the correlated pair, then the fit parameter increases by $25 \%$ and so does the free proton plus neutron EMC effect. Arrington et al. claim that if we also consider including the isospin pair counting correction and alternative fitting methods, then the range of fits expands to $0.59 \leq a \leq 1.04$. The effect of these uncertainties on the extraction of the free neutron structure function and the $d / u$ ratio at large $x_{p}$ are discussed in Ref ${ }^{75}$

\subsection{Mean-Field versus Short Ranged Correlation contributions to the EMC Effect}

We want to know whether the linear relation between the EMC slope and the SRC plateau parameter $a_{2}(A / d)$ is more than a coincidence. Any of the nuclear models discussed in Sec. 3.1 has correlations that would yield a value of $a_{2}(A / d)$ roughly consistent with the measured values. None of these models incorporate quark modifications of nuclear structure. Therefore existence of $N N$ correlations is not a sufficient condition for the EMC effect to occur. The key questions are whether the quarks confined in the two nucleons in an SRC pair have different distribution functions than those of two free nucleons. Thus the minimum input necessary to test the existence of a relation between SRC and EMC is a model of a modified twonucleon structure function consistent with a good nuclear model of SRC and with the EMC data. Here we make a first attempt at providing a link between SRC and EMC, by seeing if a modified two-nucleon structure function associated with the short-range-correlations can be used to describe deep inelastic scattering on nuclei. We also consider the other possibility, that medium modifications associated with the mean-field aspect of nuclei can describe nuclear DIS.

The treatment of Frankfurt \& Strikman (FS ${ }^{31}$ is very useful for such an aim because the nuclear structure information needed to compute deep inelastic scattering is encoded in only three integrals that can be evaluated reliably. FS derive a convolution formula

$$
\frac{1}{A} F_{2}^{A}\left(x_{A}, Q^{2}\right)=\int_{0}^{A} \alpha \rho_{A}(\alpha) F_{2}^{N}\left(x_{A} / \alpha, Q^{2}\right) d \alpha,
$$

where $\alpha \equiv \frac{A k \cdot q}{p_{A} \cdot q}$ is the fraction of the plus component of the nucleon momentum, with $k$ the struck nucleon initial momentum and $p_{A}=\left(m_{A}, 0\right)$ is the nucleus 4-momentum. $\rho_{A}(\alpha)$ is the probability that a nucleon in the nucleus carries momentum fraction $\alpha$ and $F_{2}^{N}$ is the free nucleon structure function $\left(F_{2}^{N}=\frac{1}{2}\left(F_{2}^{p}+F_{2}^{n}\right)\right)$.

Specifically $\rho(\alpha)$ is computed from the non-relativistic structure function, $S_{A, N R}(k)$

$$
\rho_{A}(\alpha)=\int d^{4} k S_{A, N R}(k) \delta\left(\alpha-\frac{k^{0}+k^{3}}{m_{N}}\right),
$$

where

$$
S_{A, \mathrm{NR}}(k, E) \equiv\left\langle A\left|a_{k}^{\dagger} \delta(E-H) a_{k}\right| A\right\rangle .
$$


The function $\rho(\alpha)$ is narrowly peaked about unity, so FS expand the nucleon structure function appearing in Eq. (10) about $\alpha=1$ to find:

$$
\frac{1}{A} F_{2}^{A}\left(x_{A}\right) \approx F_{2}^{N}\left(x_{A}\right) I_{1}(A)+x_{A}{F_{2}^{\prime N}}^{\prime} I_{2}(A)+\left[x_{A} F_{2}^{\prime N}+\frac{1}{2} x_{A}^{2} F_{2}^{\prime \prime N}\right] I_{3}(A),
$$

where for simplicity we neglect the $Q^{2}$ term in the structure function notation. The integrals $I_{n}(A)$ are given by

$$
I_{n}(A) \equiv \int \rho_{A}(\alpha) \alpha(1-\alpha)^{n-1} d \alpha, n=1,2,3
$$

Note that $I_{1}(A)=1$, which is the normalization condition.

FS proceed (see alsd ${ }^{32}$ ) by isolating the leading relativistic corrections of order $\epsilon_{A} / m$ and $k^{2} / m^{2}$ and then using the Koltun sum rule ${ }^{76}$ to find

$$
\begin{aligned}
& n_{A}(k) \equiv\left\langle A\left|a_{k}^{\dagger} a_{k}\right| A\right\rangle, I_{1}(A)=\int d^{3} k n_{A}(k) . \\
& I_{2}(A)=\int d^{3} k n_{A}(k)\left(2 \epsilon_{A} / m+\frac{A-4}{A-1} k^{2} / 6 m^{2}\right) \equiv \frac{2 \epsilon_{A}}{m}+\frac{A-4}{A-1}\left\langle\frac{k^{2}}{6 m^{2}}\right\rangle, \\
& I_{3}(A)=\int d^{3} k n_{A}(k) k^{2} / 3 m^{2}=\left\langle\frac{k^{2}}{3 m^{2}}\right\rangle .
\end{aligned}
$$

FS used the above formalism to show that a nucleons-only model without modified structure functions could not reproduce the deep inelastic scattering data.

To proceed with the calculation, we need a model of $n_{A}(k)$. The model of Ciofi degli Atti \& Simula $\sqrt{63}$ is ideal for our purposes. This is based on a realistic nuclear calculation of the spectral function that leads to nuclear densities that yield qualitative agreement with quasi elastic electron scattering. The model yields reasonably good agreement with the plateau values $a_{2}(A / d)$. Furthermore the contributions of the mean-field and correlation terms are enumerated in terms of the intermediatestate energy $E$ appearing in the spectral function of Eq. (12). This association with continuum energies, $E$, above about $20 \mathrm{MeV}$ with short range correlations is approximate but sufficiently accurate for the present schematic calculation. The spectral function leads to the momentum probability $n_{A}(k)$ such that

$$
n_{A}(k)=n_{A}^{(0)}(k)+n_{A}^{(1)}(k),
$$

where the superscript 0 refers to that obtained from low energy terms dominated by the nuclear mean field and the superscript 1 refers to high energy terms (above the continuum threshold) dominated by the effects of nucleon-nucleon correlations. Ciofi degli Atti \& Simula provide functional forms for $n_{A}(k)$ for several different nuclei. This separation using the excitation energy is not exactly the same as a separation in terms of relative momentum but is qualitatively reasonable. With this separation, terms involving correlations have about $20 \%$ of the probability.

Using Eq. (18) one can obtain the separate contributions to $I_{n}(A)$ as $I_{n}(A)=$ $I_{n}^{(0)}(A)+I_{n}^{(1)}(A)$. 


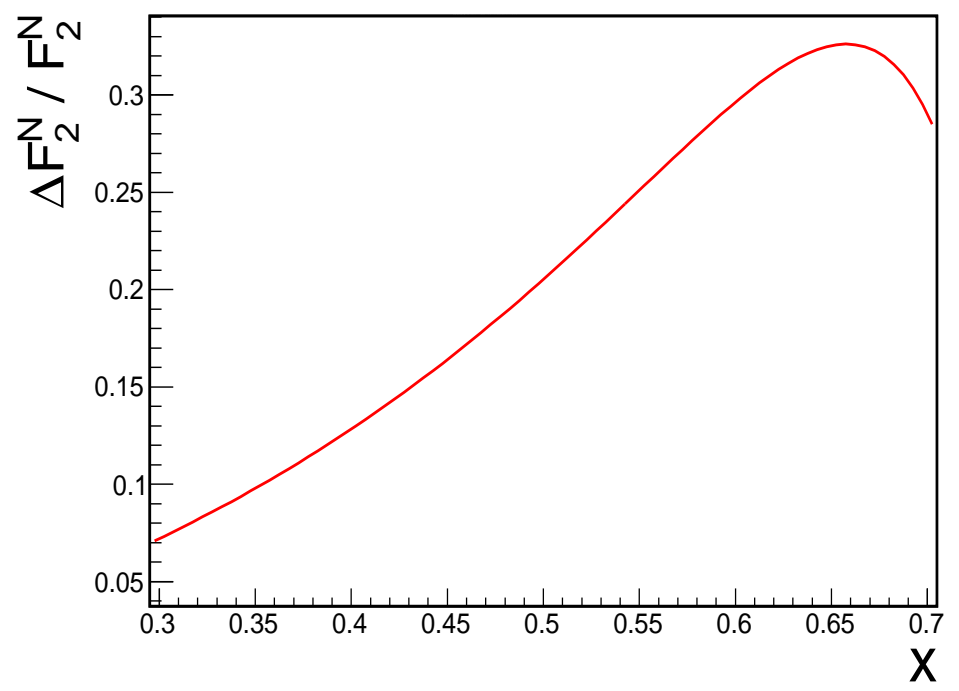

Fig. 8: The ratio of the modification term, $\Delta F_{2}^{N}$ to the free nucleon structure function, $F_{2}^{N}$.

We next proceed by assuming that nucleons in high energy excited states (correlated nucleons) have a different structure function $\widetilde{F}_{2 N}(x)$ than free ones $F_{2 N}(x)$. Thus we make the replacements

$$
I_{1}(A) F_{2} \rightarrow I_{1}^{(0)}(A) F_{2}^{N}+I_{1}^{(1)}(A) \widetilde{F}_{2}^{N}=I_{1}^{(0+1)}(A) F_{2}^{N}+I_{1}^{(1)}(A) \Delta F_{2 N}, \text { etc, }
$$

where

$$
\Delta F_{2}^{N}\left(x_{A}\right)=\widetilde{F}_{2}^{N}\left(x_{A}\right)-F_{2}^{N}\left(x_{A}\right) .
$$

An alternate version in which the medium modification is associated with the meanfield components of the density can be obtained by using

$$
I_{1}(A) F_{2}^{N} \rightarrow I_{1}^{(0)}(A) \widetilde{F}_{2}^{N}+I_{1}^{(1)}(A)=I_{1}(A) F_{2}^{N}+I_{1}^{(0)}(A) \Delta F_{2}^{N}, \text { etc. }
$$

A condition on $\Delta F_{2}^{N}$ derived from the baryon sum rule is that $\int_{0}^{2} d x_{A} \frac{\Delta F_{2}^{N}\left(x_{A}\right)}{x_{A}}=0$. This means that $\Delta F_{2}^{N}$ must pass through 0 at some value of $x_{A}$.

The analysis proceeds by calculating Eq. 13 with the supplement of Eq. 19 (Eq. 21 for the case of Mean-Field modification), assuming $\Delta F_{2}^{N}\left(x_{A}\right)$ is a second order polynomial in $x_{A}$. The parameters of $\Delta F_{2}^{N}\left(x_{A}\right)$ are fitted to the $x_{A}$ corrected EMC data (see Appendix A) for all nuclei for which momentum distributions are available (i.e., ${ }^{4} \mathrm{He},{ }^{12} \mathrm{C},{ }^{40} \mathrm{Ca},{ }^{56} \mathrm{Fe}$, and ${ }^{197} \mathrm{Au}$ ). Note that the functional form of $\Delta F_{2}^{N}\left(x_{A}\right)$ is assumed to be independent of $A$.

The results of the fits for individual nuclei are shown in Fig. 9 (Fig. 11 for the case of Mean-Field modification). The description of the data is very good for all 

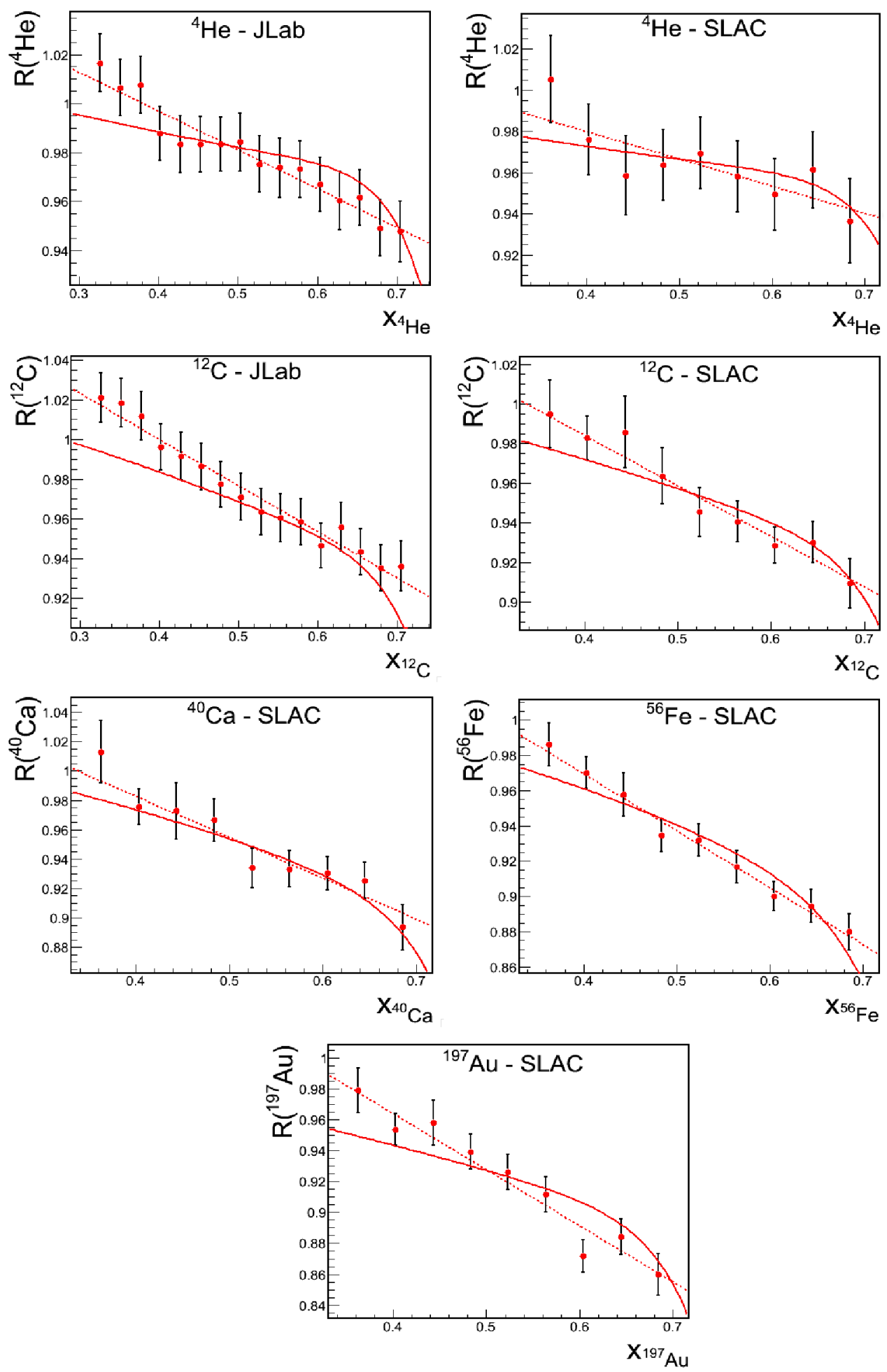

Fig. 9: The ratios of free to bound structure functions for various nuclei, extracted in the nucleus reference frame as detailed in Eq. 9. The dashed line is the result of a linear fit to the data. The solid red line is the result of the medium-modification fit, assuming an $A$-independent modification to SRC nucleons. 
nuclei with a $\chi^{2}$ per degree of freedom of $\approx 1$ for both the SRC and Mean-Field fits. These results were obtained using the parametrization of Ref ${ }^{21}$ for the free-nucleon structure function, $F_{2}^{N}$. The modified-to-free structure function ratio is shown in Fig. 8 (Fig. 10 for the case of Mean-Field modification).

The present results show that a model incorporating either universal modification of Mean-Field nucleons or modification of nucleons in SRC pairs can explain the EMC effect. As expected, the required medium modification of Mean-Field nucleons is on the order of a few percent while that of SRC nucleons is a few tens of percent. This model does not prove or disprove that the underlying cause of the EMC effect is the unique association with short ranged correlations. Note that ${ }^{9} \mathrm{Be}$ was not included in the model calculations since a ${ }^{9} \mathrm{Be}$ spectral function was not available. Note also that this model does not separate valence and sea quark distributions and therefore can't make predictions about the Drell-Yan data.

Further experiments are needed to determine whether the Mean-Field or SRC nucleons are modified by the nuclear medium. For example, quasi-elastic electron scattering would be sensitive to the former but not the latter.

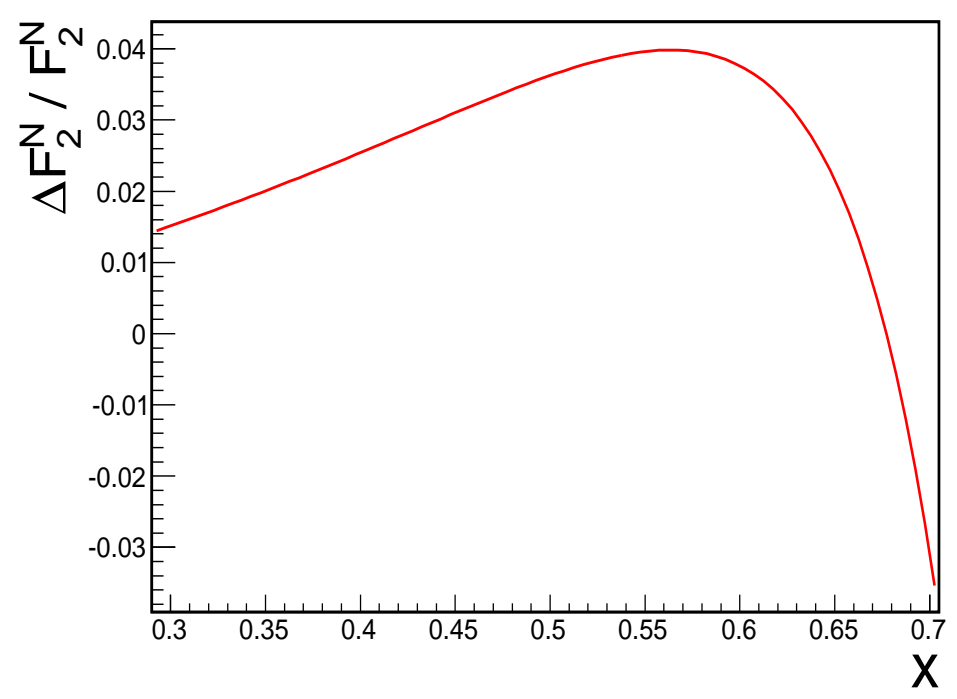

Fig. 10: Same as Fig. 8, assuming universal modification to Mean-Field nucleons. It is assumed that deuterium has no Mean-Field component, see text for details

\section{Summary}

We have reviewed recent data showing that the detailed $A$ dependence of the EMC effect provides important hints in understanding the origin of that effect. The EMC 

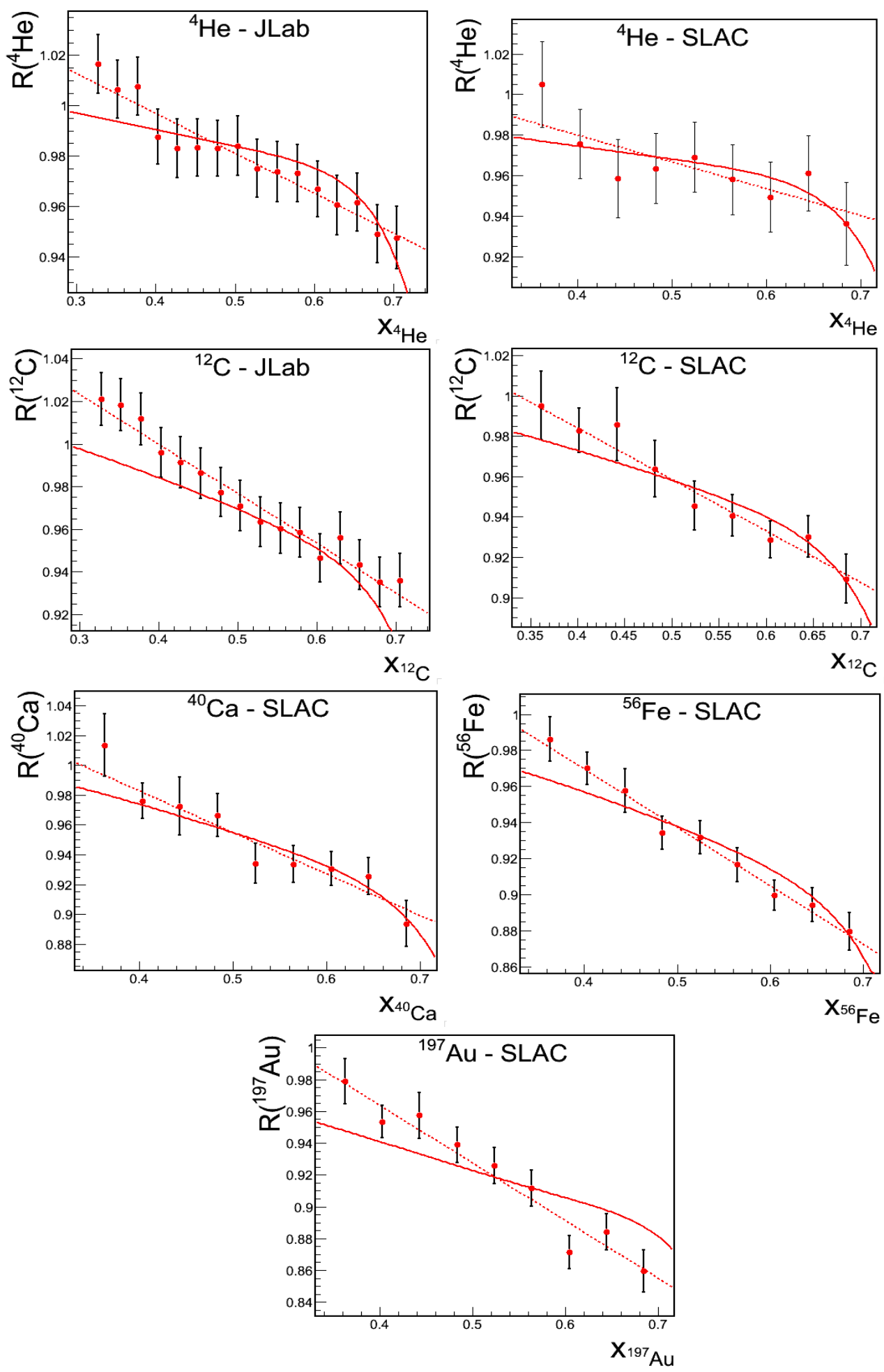

Fig. 11: Same as Fig. 9, assuming universal modification to Mean-Field nucleons. It is assumed that deuterium has no Mean-Field component, see text for details. 
effect seems to depend on local density rather than the average density. We present the EMC ratio data (the ratio of nuclear to deuterium structure functions) in terms of an improved Bjorken variable $x_{A}=A Q^{2} /\left(2 M_{A} q_{0}\right)$ (see the Appendix). We review short-range correlation data and discuss the linear relation between the EMC effect and short-range correlations. We present a phenomenological model including modification of either Mean-Field or SRC nucleons and find that modification of either is capable of accounting for the existing data.

\section{Acknowledgements}

This work is supported in part by the Israel Science Foundation, and the USIsraeli Bi-National Science Foundation, and the United States Department of Energy, Grants No. FG02-97ER41014 and DE-FG02-96ER40960.

\section{References}

1. European Muon Collaboration Collaboration (J. Aubert et al.), Phys.Lett. B123 (1983) 275.

2. A. Bodek et al., Phys.Rev.Lett 50 (1983) 14311434.

3. A. Bodek et al., Phys.Rev.Lett 51 (1983) 534537.

4. R. Arnold, P. E. Bosted, C. Chang, J. Gomez, A. Katramatou et al., Phys.Rev.Lett. 52 (1984) 727.

5. S. Dasu, P. de Barbaro, A. Bodek, H. Harada, M. Krasny et al., Phys.Rev.Lett. 60 (1988) 2591.

6. BCDMS Collaboration Collaboration (G. Bari et al.), Phys.Lett. B163 (1985) 282.

7. New Muon Collaboration Collaboration (P. Amaudruz et al.), Z.Phys. C51 (1991) 387.

8. J. Gomez, R. Arnold, P. E. Bosted, C. Chang, A. Katramatou et al., Phys.Rev. D49 (1994) 4348

9. D. F. Geesaman, K. Saito and A. W. Thomas, Ann.Rev.Nucl.Part.Sci. 45 (1995) 337.

10. R. Bickerstaff, M. Birse and G. A. Miller, Phys.Rev.Lett. 53 (1984) 2532.

11. D. Alde, H. Baer, T. Carey, G. Garvey, A. Klein et al., Phys.Rev.Lett. 64 (1990) 2479.

12. J. Seely, A. Daniel, D. Gaskell, J. Arrington, N. Fomin et al., Phys.Rev.Lett. 103 (2009) 202301.

13. S. C. Pieper and R. B. Wiringa, Ann.Rev.Nucl.Part.Sci. 51 (2001) 53.

14. J. R. Smith and G. A. Miller, Phys.Rev. C65 (2002) 055206.

15. L. Frankfurt and M. Strikman, Phys.Rept. 160 (1988) 235.

16. M. Arneodo, Phys.Rept. 240 (1994) 301.

17. G. Piller and W. Weise, Phys.Rept. 330 (2000) 1.

18. M. Sargsian, J. Arrington, W. Bertozzi, W. Boeglin, C. Carlson et al., J.Phys. G29 (2003) R1.

19. G. A. Miller, Eur.Phys.J. A31 (2007) 578.

20. R. Jaffe, Phys.Rev.Lett. 50 (1983) 228.

21. C. Carlson and T. Havens, Phys.Rev.Lett. 51 (1983) 261.

22. H. J. Pirner and J. P. Vary, Phys.Rev.Lett. 46 (1981) 1376.

23. M. Ericson and A. W. Thomas, Phys.Lett. B128 (1983) 112.

24. E. L. Berger and F. Coester, Ann.Rev.Nucl.Part.Sci. 37 (1987) 463.

25. C. Llewellyn Smith, Phys.Lett. B128 (1983) 107.

26. H. Jung and G. A. Miller, Phys.Lett. B200 (1988) 351. 
September $\quad 15, \quad 2018 \quad 9: 37 \quad$ WSPC/INSTRUCTION $\quad$ FILE EMC'reviewMar15b

27. H. Jung and A. Miller, Gerald, Phys.Rev. C41 (1990) 659.

28. R. Bickerstaff, M. Birse and G. A. Miller, Phys.Rev. D33 (1986) 3228.

29. G. A. Miller, Nuclear wave functions in deep inelastic scattering and drell-yan processes, in Nuclear and Particle Physics on the Light Cone, eds. M. B. Johnson and L. S. Kisslinger (World Scientific Press, 1988).

30. L. Frankfurt and M. Strikman, Nucl.Phys. B316 (1989) 340.

31. L. Frankfurt and M. Strikman, Phys.Lett. B183 (1987) 254.

32. C. Ciofi degli Atti and S. Liuti, Phys.Rev. C44 (1991) 1269.

33. A. Dieperink and G. A. Miller, Phys.Rev. C44 (1991) 866.

34. L. Frankfurt and M. Strikman, Int.J.Mod.Phys. E21 (2012) 1230002.

35. G. A. Miller and J. R. Smith, Phys.Rev. C65 (2002) 015211.

36. H. Mineo, W. Bentz, N. Ishii, A. W. Thomas and K. Yazaki, Nucl.Phys. A735 (2004) 482.

37. I. Cloet, W. Bentz and A. W. Thomas, Phys.Lett. B642 (2006) 210.

38. J. R. Smith and G. A. Miller, Phys.Rev.Lett. 91 (2003) 212301.

39. L. Frankfurt and M. Strikman, Nucl.Phys. B250 (1985) 143.

40. M. Frank, B. Jennings and G. A. Miller, Phys.Rev. C54 (1996) 920.

41. S. A. Kulagin and R. Petti, Nucl.Phys. A765 (2006) 126.

42. F. Halzen and A. D. Martin, Quarks and Leptons: An Introductory Course in Modern Particle Physics (Wiley, New York, USA, 1984).

43. L. W. Whitlow, E. M. Riordan, S. Dasu, S. Rock and A. Bodek, Phys.Lett. B282 (1992) 475482.

44. P. E. Bosted and M. E. Christy, Phys.Rev. C77 (2008) 065206.

45. A. Bodek et al., Phys.Rev. D20 (1979) 14711552.

46. J. Arrington, F. Coester, R. J. Holt and T.-S. H. Lee, J. Phys. G: Nucl. Part. Phys. 36 (2009) 025005.

47. H. Bethe, Ann.Rev.Nucl.Part.Sci. 21 (1971) 93.

48. M. Anastasio, L. Celenza, W. Pong and C. Shakin, Phys.Rept. 100 (1983) 327.

49. R. Brockmann and R. Machleidt, Phys.Lett. B149 (1984) 283.

50. G. A. Miller and R. Machleidt, Phys.Rev. C60 (1999) 035202.

51. G. A. Miller, Prog.Part.Nucl.Phys. 45 (2000) 83.

52. P. F. Bedaque and U. van Kolck, Ann.Rev.Nucl.Part.Sci. 52 (2002) 339.

53. S. Bogner, R. Furnstahl and A. Schwenk, Prog.Part.Nucl.Phys. 65 (2010) 94.

54. J. Kelly, Adv.Nucl.Phys. 23 (1996) 75.

55. CLAS Collaboration (K. Egiyan et al.), Phys.Rev. C68 (2003) 014313.

56. L. L. Frankfurt, M. M. Sargsian and M. I. Strikman, Phys.Rev. C56 (1997) 1124.

57. M. M. Sargsian, Int.J.Mod.Phys. E10 (2001) 405.

58. R. G. Arnold et al., Phys.Rev. C42 (1990) R1.

59. M. J. Laget et al., Phys.Lett. B199 (1987) 493.

60. L. Frankfurt, M. Strikman, D. Day and M. Sargsian, Phys.Rev. C48 (1993) 2451.

61. CLAS Collaboration (K. Egiyan et al.), Phys.Rev.Lett 96 (2006) 082501.

62. N. Fomin et al., Phys.Rev.Lett 108 (2012) 092502.

63. C. Ciofi degli Atti and S. Simula, Phys.Rev. C53 (1996) 1689.

64. A. Tang, J. W. Watson, J. Aclander et al., Phys.Rev.Lett. 90 (2003) 042301.

65. E. Piasetzky, M. Sargsian, L. Frankfurt, M. Strikman and J. Watson, Phys.Rev.Lett. 97 (2006) 162504.

66. R. Shneor, P. Monaghan, R. Subedi et al., Phys.Rev.Lett. 99 (2007) 072501.

67. R. Subedi, R. Shneor, P. Monaghan et al., Science 320 (2008) 1476.

68. M. Sargsian, T. Abrahamyan, M. Strikman and L. Frankfurt, Phys.Rev. C71 (2005) 044615 . 
September EMC'reviewMar15b

69. R. Schiavilla, R. B. Wiringa, S. C. Pieper and J. Carlson, Phys.Rev.Lett. 98 (2007) 132501.

70. L. B. Weinstein, E. Piasetzky, D. W. Higinbotham, J. Gomez, O. Hen and R. Shneor, Phys. Rev. Lett. 106 (Feb 2011) 052301.

71. O. Hen, E. Piasetzky and L. B. Weinstein, Phys. Rev. C 85 (Apr 2012) 047301.

72. J. Arrington, A. Daniel, D. Day, N. Fomin, D. Gaskell et al., Phys.Rev. C86 (2012) 065204 .

73. A. Accardi, W. Melnitchouk, J. Owens, M. Christy, C. Keppel et al., Phys.Rev. D84 (2011) 014008.

74. O. Hen, A. Accardi, W. Melnitchouk and E. Piasetzky, Phys. Rev. D 84 (2011) 117501.

75. E. Piasetzky, O. Hen and L. Weinstein, arXiv: 1209.0636 (2012).

76. D. Koltun, Phys.Rev.Lett. 28 (1972) 182.

\section{Appendix A. EMC Data Base}

In this appendix we present a new data-base for the structure function ratio of nuclei $\left(F_{2}^{A}\right)$ relative to deuterium $\left(F_{2}^{d}\right)$. The ratio is taken using $F_{2}^{A}$ and $F_{2}^{d}$ each extracted in its own reference frame, at equivalent kinematical regions, defined by $x_{A}$ and $Q^{2}$. The extraction of the structure function follows the formalism of section 2.3] and is shown only for the range of $0.3 \leq x_{A} \leq 0.7$.

We use JLab and SLAC high precision data on DIS cross section ratios for nuclei relative to deuterium as input to Eq. 9$]^{[12]}$ We use a parametrization of $F_{2}^{d}\left(x, Q^{2}\right)$ from re ${ }^{44}$ to move the deuteron measurement to $x_{A}$, and reapply isoscalar corrections using a parametrization of $R_{n p}\left(x_{A}, Q^{2}\right)=F_{2}^{n}\left(x_{A}, Q^{2}\right) / F_{2}^{p}\left(x_{A}, Q^{2}\right)$ from ref. The results are presented in tables 18 are for the SLAC data and tables 9 - 12 are for the JLab data. Note that the SLAC results are averaged over $Q^{2}=2,5$, and 10 $\mathrm{GeV} / \mathrm{c}^{2}$.

Table 1: EMC data for ${ }^{4} \mathrm{He}$ from SLAC. The left side of the table shows the original as published data from table VIII of ref $\frac{8}{8}$ The right side is the same data, corrected for the definition of $x_{A}$ according to Eq. 9 .

\begin{tabular}{|c|c|c||c|c|c|}
\hline $\mathrm{x}$ & $F_{2}^{4} H e / F_{2}^{d}(x)$ & $\Delta F_{2}^{4} H e / F_{2}^{d}(x)$ & $x_{A}$ & $F_{2}^{4} H e / F_{2}^{d}\left(x_{A}\right)$ & $\Delta F_{2}^{4} H e / F_{2}^{d}\left(x_{A}\right)$ \\
\hline 0.36 & 0.998 & 0.021 & 0.362 & 1.005 & 0.021 \\
0.40 & 0.968 & 0.017 & 0.402 & 0.976 & 0.017 \\
0.44 & 0.949 & 0.019 & 0.443 & 0.958 & 0.019 \\
0.48 & 0.954 & 0.017 & 0.483 & 0.963 & 0.017 \\
0.52 & 0.951 & 0.017 & 0.523 & 0.969 & 0.017 \\
0.56 & 0.943 & 0.017 & 0.563 & 0.958 & 0.017 \\
0.60 & 0.928 & 0.017 & 0.604 & 0.949 & 0.017 \\
0.64 & 0.935 & 0.018 & 0.644 & 0.961 & 0.018 \\
0.68 & 0.917 & 0.020 & 0.684 & 0.936 & 0.020 \\
\hline
\end{tabular}


September $\quad 2018 \quad 9: 37 \quad$ WSPC/INSTRUCTION $\quad$ FILE

The EMC Effect and High Momentum Nucleons in Nuclei

Table 2: Same as table1, for ${ }^{9} \mathrm{Be}$.

\begin{tabular}{|c|c|c|c|c|c|}
\hline $\mathrm{x}$ & $F_{2}^{9} B e / F_{2}^{d}(x)$ & $\Delta F_{2}^{9} B e / F_{2}^{d}(x)$ & $x_{A}$ & $F_{2}^{9} B e / F_{2}^{d}\left(x_{A}\right)$ & $\Delta F_{2}^{9} B e / F_{2}^{d}\left(x_{A}\right)$ \\
\hline 0.36 & 0.993 & 0.014 & 0.362 & 1.007 & 0.014 \\
\hline 0.40 & 0.957 & 0.009 & 0.402 & 0.972 & 0.009 \\
\hline 0.44 & 0.980 & 0.014 & 0.443 & 0.997 & 0.014 \\
\hline 0.48 & 0.951 & 0.011 & 0.483 & 0.968 & 0.011 \\
\hline 0.52 & 0.955 & 0.011 & 0.523 & 0.979 & 0.011 \\
\hline 0.56 & 0.945 & 0.011 & 0.563 & 0.967 & 0.011 \\
\hline 0.60 & 0.928 & 0.010 & 0.604 & 0.955 & 0.010 \\
\hline 0.64 & 0.917 & 0.011 & 0.644 & 0.947 & 0.011 \\
\hline 0.68 & 0.912 & 0.013 & 0.684 & 0.935 & 0.013 \\
\hline
\end{tabular}

Table 3: Same as table [1, for ${ }^{12} \mathrm{C}$.

\begin{tabular}{|c|c|c||c|c|c|}
\hline $\mathrm{x}$ & $F_{2}^{12} C / F_{2}^{d}(x)$ & $\Delta F_{2}^{12} C / F_{2}^{d}(x)$ & $x_{A}$ & $F_{2}^{12} C / F_{2}^{d}\left(x_{A}\right)$ & $\Delta F_{2}^{12} C / F_{2}^{d}\left(x_{A}\right)$ \\
\hline 0.36 & 0.987 & 0.017 & 0.362 & 0.995 & 0.017 \\
0.40 & 0.974 & 0.011 & 0.403 & 0.983 & 0.011 \\
0.44 & 0.975 & 0.018 & 0.443 & 0.986 & 0.018 \\
0.48 & 0.953 & 0.014 & 0.483 & 0.963 & 0.014 \\
0.52 & 0.926 & 0.012 & 0.523 & 0.945 & 0.012 \\
0.56 & 0.924 & 0.010 & 0.564 & 0.940 & 0.010 \\
0.60 & 0.905 & 0.009 & 0.604 & 0.928 & 0.009 \\
0.64 & 0.903 & 0.010 & 0.644 & 0.930 & 0.010 \\
0.68 & 0.888 & 0.012 & 0.685 & 0.909 & 0.012 \\
\hline
\end{tabular}

Table 4: Same as table[1, for ${ }^{27} \mathrm{Al}$.

\begin{tabular}{|c|c|c||c|c|c|}
\hline $\mathrm{x}$ & $F_{2}^{27} A l / F_{2}^{d}(x)$ & $\Delta F_{2}^{27} A l / F_{2}^{d}(x)$ & $x_{A}$ & $F_{2}^{27} A l / F_{2}^{d}\left(x_{A}\right)$ & $\Delta F_{2}^{27} A l / F_{2}^{d}\left(x_{A}\right)$ \\
\hline 0.36 & 0.993 & 0.013 & 0.362 & 1.005 & 0.013 \\
0.40 & 0.966 & 0.009 & 0.403 & 0.977 & 0.009 \\
0.44 & 0.959 & 0.012 & 0.443 & 0.973 & 0.012 \\
0.48 & 0.934 & 0.010 & 0.483 & 0.948 & 0.010 \\
0.52 & 0.926 & 0.010 & 0.524 & 0.950 & 0.010 \\
0.56 & 0.923 & 0.009 & 0.564 & 0.944 & 0.009 \\
0.60 & 0.906 & 0.009 & 0.604 & 0.934 & 0.009 \\
0.64 & 0.892 & 0.009 & 0.645 & 0.923 & 0.009 \\
0.68 & 0.876 & 0.011 & 0.685 & 0.900 & 0.011 \\
\hline
\end{tabular}


September EMC'reviewMar15b

FILE

Table 5: Same as table 1, for ${ }^{40} \mathrm{Ca}$.

\begin{tabular}{|c|c|c||c|c|c|}
\hline $\mathrm{x}$ & $F_{2}^{40} \mathrm{Ca} / F_{2}^{d}(x)$ & $\Delta F_{2}^{40} \mathrm{Ca} / F_{2}^{d}(x)$ & $x_{A}$ & $F_{2}^{40} \mathrm{Ca} / F_{2}^{d}\left(x_{A}\right)$ & $\Delta F_{2}^{40} \mathrm{Ca} / F_{2}^{d}\left(x_{A}\right)$ \\
\hline 0.36 & 1.004 & 0.021 & 0.363 & 1.013 & 0.021 \\
0.40 & 0.966 & 0.012 & 0.403 & 0.975 & 0.012 \\
0.44 & 0.960 & 0.019 & 0.443 & 0.972 & 0.019 \\
0.48 & 0.954 & 0.014 & 0.484 & 0.966 & 0.014 \\
0.52 & 0.912 & 0.013 & 0.524 & 0.934 & 0.013 \\
0.56 & 0.915 & 0.012 & 0.564 & 0.933 & 0.012 \\
0.60 & 0.904 & 0.011 & 0.605 & 0.930 & 0.011 \\
0.64 & 0.895 & 0.012 & 0.645 & 0.925 & 0.012 \\
0.68 & 0.870 & 0.015 & 0.685 & 0.893 & 0.015 \\
\hline
\end{tabular}

Table 6: Same as table 1 for ${ }^{56} \mathrm{Fe}$.

\begin{tabular}{|c|c|c||c|c|c|}
\hline $\mathrm{x}$ & $F_{2}^{56} \mathrm{Fe} / F_{2}^{d}(x)$ & $\Delta F_{2}^{56} \mathrm{Fe} / F_{2}^{d}(x)$ & $x_{A}$ & $F_{2}^{56} \mathrm{Fe} / F_{2}^{d}\left(x_{A}\right)$ & $\Delta F_{2}^{56} \mathrm{Fe} / F_{2}^{d}\left(x_{A}\right)$ \\
\hline 0.36 & 0.972 & 0.012 & 0.363 & 0.986 & 0.012 \\
0.40 & 0.955 & 0.009 & 0.403 & 0.970 & 0.009 \\
0.44 & 0.940 & 0.012 & 0.443 & 0.957 & 0.012 \\
0.48 & 0.917 & 0.009 & 0.484 & 0.934 & 0.009 \\
0.52 & 0.904 & 0.009 & 0.524 & 0.931 & 0.009 \\
0.56 & 0.893 & 0.009 & 0.564 & 0.916 & 0.009 \\
0.60 & 0.869 & 0.008 & 0.605 & 0.899 & 0.008 \\
0.64 & 0.860 & 0.009 & 0.645 & 0.894 & 0.009 \\
0.68 & 0.852 & 0.010 & 0.685 & 0.879 & 0.010 \\
\hline
\end{tabular}

Table 7: Same as table 1, for ${ }^{108} \mathrm{Ag}$.

\begin{tabular}{|c|c|c||c|c|c|}
\hline $\mathrm{x}$ & $F_{2}{ }^{\mathrm{I08}} A g / F_{2}^{d}(x)$ & $\Delta F_{2}^{108} A g / F_{2}^{d}(x)$ & $x_{A}$ & $F_{2}^{108} A g / F_{2}^{d}\left(x_{A}\right)$ & $\Delta F_{2}^{\text {I08 } A g} / F_{2}^{d}\left(x_{A}\right)$ \\
\hline 0.36 & 1.012 & 0.023 & 0.363 & 1.031 & 0.023 \\
0.40 & 0.968 & 0.013 & 0.403 & 0.988 & 0.013 \\
0.44 & 0.957 & 0.021 & 0.443 & 0.979 & 0.021 \\
0.48 & 0.926 & 0.015 & 0.484 & 0.948 & 0.015 \\
0.52 & 0.897 & 0.014 & 0.524 & 0.928 & 0.014 \\
0.56 & 0.891 & 0.013 & 0.564 & 0.918 & 0.013 \\
0.60 & 0.881 & 0.012 & 0.605 & 0.915 & 0.012 \\
0.64 & 0.842 & 0.013 & 0.645 & 0.878 & 0.013 \\
0.68 & 0.842 & 0.016 & 0.685 & 0.871 & 0.016 \\
\hline
\end{tabular}


September EMC'reviewMar15b

2018 9:37 WSPC/INSTRUCTION

FILE

Table 8: Same as table 1, for ${ }^{197} \mathrm{Au}$.

\begin{tabular}{|c|c|c||c|c|c|}
\hline $\mathrm{x}$ & $F_{2}^{197} A u / F_{2}^{d}(x)$ & $\Delta F_{2}^{197} A u / F_{2}^{d}(x)$ & $x_{A}$ & $F_{2}^{197} A u / F_{2}^{d}\left(x_{A}\right)$ & $\Delta F_{2}^{197} A u / F_{2}^{d}\left(x_{A}\right)$ \\
\hline 0.36 & 0.956 & 0.014 & 0.362 & 0.979 & 0.014 \\
0.40 & 0.930 & 0.010 & 0.403 & 0.953 & 0.010 \\
0.44 & 0.931 & 0.014 & 0.443 & 0.957 & 0.014 \\
0.48 & 0.914 & 0.011 & 0.483 & 0.939 & 0.011 \\
0.52 & 0.892 & 0.011 & 0.524 & 0.926 & 0.011 \\
0.56 & 0.881 & 0.011 & 0.564 & 0.911 & 0.011 \\
0.60 & 0.837 & 0.010 & 0.604 & 0.871 & 0.010 \\
0.64 & 0.846 & 0.011 & 0.644 & 0.884 & 0.011 \\
0.68 & 0.829 & 0.013 & 0.685 & 0.859 & 0.013 \\
\hline
\end{tabular}

Table 9: EMC data for ${ }^{3} \mathrm{He}$ from JLab. The left side of the table shows the original as published data from ref. ${ }^{12}$ The right side is the same data, corrected for the definition of $x_{A}$ according to Eq. 9 .

\begin{tabular}{|c|c|c||c|c|c|}
\hline $\mathrm{x}$ & $F_{2}^{{ }^{3} \mathrm{He}} / F_{2}^{d}(x)$ & $\Delta F_{2}^{{ }^{3} \mathrm{He}} / F_{2}^{d}(x)$ & $x_{A}$ & $F_{2}{ }^{3} \mathrm{He} / F_{2}{ }^{d}\left(x_{A}\right)$ & $\Delta F_{2}^{{ }^{3} \mathrm{He}} / F_{2}^{d}\left(x_{A}\right)$ \\
\hline 0.325 & 0.9774 & 0.011453 & 0.325 & 0.970073 & 0.0113672 \\
0.350 & 0.9763 & 0.0113158 & 0.350 & 0.968431 & 0.0112246 \\
0.375 & 0.9796 & 0.0113219 & 0.375 & 0.971228 & 0.0112251 \\
0.400 & 0.9684 & 0.0107239 & 0.400 & 0.959746 & 0.0106281 \\
0.425 & 0.9725 & 0.0114144 & 0.425 & 0.963504 & 0.0113089 \\
0.450 & 0.9713 & 0.0112523 & 0.450 & 0.961924 & 0.0111437 \\
0.475 & 0.9696 & 0.0108533 & 0.476 & 0.960588 & 0.0107524 \\
0.500 & 0.9629 & 0.0114935 & 0.501 & 0.95354 & 0.0113818 \\
0.525 & 0.9599 & 0.0112036 & 0.526 & 0.949848 & 0.0110863 \\
0.550 & 0.964 & 0.0118444 & 0.551 & 0.955055 & 0.0117345 \\
0.575 & 0.9653 & 0.0113391 & 0.576 & 0.955923 & 0.0112289 \\
0.600 & 0.9644 & 0.0109817 & 0.601 & 0.954435 & 0.0108683 \\
0.625 & 0.949 & 0.0118153 & 0.626 & 0.94051 & 0.0117096 \\
0.650 & 0.9611 & 0.0115051 & 0.651 & 0.951985 & 0.0113959 \\
0.675 & 0.9562 & 0.0116562 & 0.676 & 0.945647 & 0.0115276 \\
0.700 & 0.9479 & 0.0125035 & 0.701 & 0.938561 & 0.0123803 \\
\hline
\end{tabular}


September $\quad 2018 \quad 9: 37 \quad$ WSPC/INSTRUCTION $\quad$ FILE EMC'reviewMar15b

Table 10: Same as table 9, for ${ }^{4} \mathrm{He}$.

\begin{tabular}{|c|c|c||c|c|c|}
\hline $\mathrm{x}$ & $F_{2}^{4} \mathrm{He} / F_{2}^{d}(x)$ & $\Delta F_{2}^{4} \mathrm{He} / F_{2}^{d}(x)$ & $x_{A}$ & $F_{2}^{4} \mathrm{He} / F_{2}^{d}\left(x_{A}\right)$ & $\Delta F_{2}^{4} \mathrm{He} / F_{2}^{d}\left(x_{A}\right)$ \\
\hline 0.325 & 1.011 & 0.0116698 & 0.327 & 1.01653 & 0.0117336 \\
0.350 & 0.9998 & 0.0114398 & 0.352 & 1.00644 & 0.0115158 \\
0.375 & 0.9996 & 0.0114471 & 0.377 & 1.00753 & 0.0115379 \\
0.400 & 0.9784 & 0.0108174 & 0.402 & 0.987558 & 0.0109187 \\
0.425 & 0.9727 & 0.0114294 & 0.427 & 0.983079 & 0.0115513 \\
0.450 & 0.9724 & 0.0112776 & 0.453 & 0.98331 & 0.0114042 \\
0.475 & 0.9688 & 0.0108597 & 0.478 & 0.983158 & 0.0110206 \\
0.500 & 0.9695 & 0.0115953 & 0.503 & 0.984013 & 0.0117688 \\
0.525 & 0.9613 & 0.0112505 & 0.528 & 0.975085 & 0.0114118 \\
0.550 & 0.955 & 0.0117935 & 0.553 & 0.973823 & 0.0120259 \\
0.575 & 0.9542 & 0.0112231 & 0.578 & 0.973198 & 0.0114466 \\
0.600 & 0.9491 & 0.0107922 & 0.604 & 0.966896 & 0.0109946 \\
0.625 & 0.9361 & 0.0115938 & 0.629 & 0.960513 & 0.0118962 \\
0.650 & 0.9389 & 0.0111987 & 0.654 & 0.961687 & 0.0114705 \\
0.675 & 0.9315 & 0.0113139 & 0.679 & 0.949143 & 0.0115282 \\
0.700 & 0.9238 & 0.0121262 & 0.704 & 0.947597 & 0.0124385 \\
\hline
\end{tabular}

Table 11: Same as table 9, for ${ }^{9} \mathrm{Be}$.

\begin{tabular}{|c|c|c||c|c|c|}
\hline $\mathrm{x}$ & $F_{2}^{{ }^{9} B e} / F_{2}^{d}(x)$ & $\Delta F_{2}^{{ }^{9} B e} / F_{2}^{d}(x)$ & $x_{A}$ & $F_{2}^{{ }^{9} B e} / F_{2}^{d}\left(x_{A}\right)$ & $\Delta F_{2}^{{ }^{9} B e} / F_{2}^{d}\left(x_{A}\right)$ \\
\hline 0.325 & 1.027 & 0.0134537 & 0.327 & 1.03628 & 0.0135753 \\
0.350 & 1.018 & 0.0131816 & 0.352 & 1.02794 & 0.0133104 \\
0.375 & 1.014 & 0.0130497 & 0.377 & 1.02575 & 0.0132009 \\
0.400 & 0.9977 & 0.0124262 & 0.402 & 1.0112 & 0.0125944 \\
0.425 & 0.9907 & 0.0127738 & 0.427 & 1.00495 & 0.0129575 \\
0.450 & 0.9821 & 0.0124847 & 0.452 & 0.997484 & 0.0126803 \\
0.475 & 0.9745 & 0.0120235 & 0.477 & 0.992554 & 0.0122463 \\
0.500 & 0.9709 & 0.012429 & 0.503 & 0.989694 & 0.0126696 \\
0.525 & 0.9567 & 0.0119992 & 0.528 & 0.974422 & 0.0122215 \\
0.550 & 0.9538 & 0.0123411 & 0.553 & 0.976809 & 0.0126388 \\
0.575 & 0.9469 & 0.0118303 & 0.578 & 0.969655 & 0.0121146 \\
0.600 & 0.9403 & 0.0114402 & 0.603 & 0.961711 & 0.0117006 \\
0.625 & 0.9459 & 0.0122301 & 0.628 & 0.974281 & 0.0125971 \\
0.650 & 0.9322 & 0.0116412 & 0.653 & 0.958901 & 0.0119747 \\
0.675 & 0.9269 & 0.0116589 & 0.679 & 0.948751 & 0.0119338 \\
0.700 & 0.9201 & 0.0122438 & 0.704 & 0.947675 & 0.0126108 \\
\hline
\end{tabular}


September EMC'reviewMar15b

2018 9:37 WSPC/INSTRUCTION

FILE

Table 12: Same as table 9, for ${ }^{12} \mathrm{C}$.

\begin{tabular}{|c|c|c||c|c|c|}
\hline $\mathrm{x}$ & $F_{2}^{12} C / F_{2}^{d}(x)$ & $\Delta F_{2}^{12} C / F_{2}^{d}(x)$ & $x_{A}$ & $F_{2}^{12} C / F_{2}^{d}\left(x_{A}\right)$ & $\Delta F_{2}^{12} C / F_{2}^{d}\left(x_{A}\right)$ \\
\hline 0.325 & 1.015 & 0.0123864 & 0.327 & 1.0211 & 0.0124609 \\
0.350 & 1.011 & 0.0122230 & 0.352 & 1.01838 & 0.0123122 \\
0.375 & 1.003 & 0.0120969 & 0.377 & 1.01175 & 0.0122024 \\
0.400 & 0.9859 & 0.0115927 & 0.403 & 0.996045 & 0.011712 \\
0.425 & 0.9798 & 0.0119255 & 0.428 & 0.991303 & 0.0120655 \\
0.450 & 0.9743 & 0.0117119 & 0.453 & 0.986333 & 0.0118566 \\
0.475 & 0.9617 & 0.0113265 & 0.478 & 0.977356 & 0.0115109 \\
0.500 & 0.9553 & 0.0117368 & 0.503 & 0.971005 & 0.0119297 \\
0.525 & 0.9485 & 0.0114379 & 0.528 & 0.963524 & 0.0116191 \\
0.550 & 0.9401 & 0.0117128 & 0.554 & 0.960463 & 0.0119665 \\
0.575 & 0.938 & 0.0113657 & 0.579 & 0.958495 & 0.0116141 \\
0.600 & 0.9274 & 0.0110039 & 0.604 & 0.946495 & 0.0112305 \\
0.625 & 0.9291 & 0.0119147 & 0.629 & 0.955914 & 0.0122586 \\
0.650 & 0.9191 & 0.0113583 & 0.654 & 0.943392 & 0.0116585 \\
0.675 & 0.9162 & 0.0114603 & 0.680 & 0.93512 & 0.011697 \\
0.700 & 0.9107 & 0.0121902 & 0.705 & 0.936063 & 0.0125297 \\
\hline
\end{tabular}

